Images du travail, travail des images

$9 \mid 2020$

L'apprentissage et l'enseignement professionnel en images

\title{
Penser les relations entre éducation et travail, l'expérience de Lucie Tanguy
}

Stéphane Lembré, Gilles Moreau et Lucie Tanguy

\section{OpenEdition}

\section{Journals}

Édition électronique

URL : http://journals.openedition.org/itti/514

DOI : 10.4000/itti.514

\section{Éditeur}

Université de Poitiers

Référence électronique

Stéphane Lembré, Gilles Moreau et Lucie Tanguy, « Penser les relations entre éducation et travail, l'expérience de Lucie Tanguy », Images du travail, travail des images [En ligne], 9 | 2020, mis en ligne le 01 septembre 2020, consulté le 14 avril 2021. URL : http://journals.openedition.org/itti/514 ; DOI : https://doi.org/10.4000/itti.514

Ce document a été généré automatiquement le 14 avril 2021

Images du travail, travail des images 


\title{
Penser les relations entre éducation et travail, l'expérience de Lucie Tanguy
}

\author{
Stéphane Lembré, Gilles Moreau et Lucie Tanguy
}

\section{NOTE DE L'AUTEUR}

Plutôt rétive à cet exercice au départ, aujourd'hui reconnaissante à Stéphane Lembré et Gilles Moreau qui m'ont convaincue de le faire et m'ont accompagnée tout au long de ce retour sur mon expérience du métier de sociologue. Je les en remercie très vivement. Merci également à Jean-Paul Géhin pour la dernière lecture et la mise en forme de ce texte en vue de sa publication.

Lucie Tanguy

1 Lucie Tanguy est directrice de recherche honoraire au CNRS. Ses travaux sur l'enseignement professionnel sont incontournables dans ce champ entre autres parce qu'elle a su y articuler sociologie du travail et sociologie de l'éducation. Elle avait donc toute sa place dans ce numéro d'Images du travail/travail des images dans la mesure où ses ouvrages, de l'enseignement professionnel en France (PUF, 1991) à Enseigner l'esprit d'entreprise à l'école (La Dispute, 2016), de même que ses nombreux articles, ont fait date et ouvert des chemins souvent en friches et/ou inexploités par l'analyse sociologique. Mais Lucie Tanguy ne s'est pas laissée enfermer dans une spécialité : elle a su aussi explorer des contrées voisines, comme en attestent Les instituts du travail : la formation syndicale à l'université de 1955 à nos jours (PUR, 2006) ou encore La sociologie du travail en France. Enquête sur le travail des sociologues (1950-1990) (La Découverte 2011). L'inviter à revenir à l'occasion de cet entretien sur son travail était donc l'occasion de comprendre comment l'esprit scientifique et critique qui l'habite et l'anime avait su s'allier dans sa démarche de recherche. 
2 Cet entretien a été réalisé entre décembre 2019 et mai 2020 par échange mail, à partir d'un jeu de questions initialement proposées. Il a été ensuite enrichi par les échanges et réactions aux versions précédentes. Les notes de bas de page ont été introduites par Lucie Tanguy, Stéphane Lembré et Gilles Moreau.

QUESTION : En exergue de ton dernier ouvrage, Enseigner l'esprit d'entreprise à l'école (2016), tu mets une dédicace à tes parents : à ta mère «bonne » et à ton père qui te voyait, après l'obtention de ton BEPC devenir «président[e] », autant de propos qui laissent entendre une origine sociale populaire. Peux-tu nous en dire plus sur ton histoire sociale et scolaire?

Cette question est très embarrassante et j'ai longtemps hésité à y répondre. Elle est devenue une sorte de rituel qui devrait être questionné. On déduit, en effet, bien trop vite de la biographie des auteurs, leur manière de faire des recherches. Aujourd'hui cette réticence s'est amenuisée avec la mort de mes parents qui a opéré une sorte de métamorphose intérieure. J'ai pris pleinement conscience que le mérite attribué à la chercheure que je suis, était avant tout le leur. Ils ont payé au prix fort les études de leurs deux filles et leur accès à des professions intellectuelles. Mon père a connu la solitude de l'ouvrier agricole émigré en Beauce comme beaucoup d'hommes issus de la petite paysannerie bretonne après la seconde guerre mondiale. Ma mère, elle aussi ouvrière agricole, a dû plus tard revenir au pays pour élever ses filles et subir des relations violentes avec des adolescentes révoltées.

Après coup, il me semble que six années d'école primaire au couvent, réglées par la soumission, m'ont rendue rebelle. Des années de collège dans une institution catholique, il me reste l'humiliation infligée par mes compagnes de classe, filles de commerçants, mieux habillées, parlant mieux que moi qui vivait à la campagne. Au lycée public de la petite ville où mes parents avaient pu acheter une maison, un professeur de physique et chimie chez qui ma mère faisait des ménages m'a (et l'a) convaincue que je devais faire des études supérieures, mais impossible sans ressources. Après le bac, devenue "pionne " j'ai voulu entreprendre des études de physique mais l'université était trop loin pour que je puisse suivre régulièrement les cours. J'ai ensuite postulé pour un poste d'institutrice et ai été nommée à Stains en région parisienne où je ne suis restée qu'un an me sentant mal à l'aise dans ce métier. Je me suis alors inscrite en littérature à la Sorbonne. Des sciences de la nature à la littérature et aux sciences sociales, le chemin est trop long et tortueux pour être raconté. Loin d'être linéaire, il a été fait de circonstances, de rencontres toutes aussi aléatoires les unes que les autres.

J'allais passer sous silence les épisodes de travail manuel pendant les vacances scolaires. Ils étaient certes nécessaires pour payer les fournitures scolaires, mais en fait ils ont donné lieu à des expériences constructives. J'ai éprouvé, en travaillant dans les fermes comme bergère d'abord et plus durement en ramassant des haricots ou des pommes de terre dans les champs et ensuite dans une usine de conserves de sardines, la résistance physique et psychique qu'exige le travail manuel. Il me semble avoir incorporé puis converti ces qualités dans celles du travail intellectuel, pour lesquels la concentration, l'immobilité du corps, l'endurance sont des apprentissages difficiles pour les enfants qui n'y ont pas été habitués (souligné par Gramsci). À ma grande surprise, un professeur d'atelier, ancien ouvrier, que j'interrogeais il y a une trentaine d'années, m'a dit «j'aimerais pas faire votre travail, rester immobile sur une chaise pour écrire !!! ». 
Au terme de ce parcours, je crois pouvoir dire qu'avoir éprouvé l'injustice sociale et l'humiliation a fortement influé sur mes engagements intellectuels et politiques. À la différence de ce que dit Pierre Bourdieu sur la « honte de soi » ressentie par celles et ceux qui ont franchi les barrières entre classes populaires et classes supérieures, je n'ai jamais éprouvé ce sentiment. En revanche, le manque de confiance en moi m'a toujours tenaillée et obligée de parer à la peur de la médiocrité, qui me hantait, par un surcroît de travail, en gardant toujours présents à l'esprit tous ceux et celles que j'avais laissés derrière moi.

QUESTION : Tu accèdes à l'enseignement supérieur à un moment où la sociologie n'est pas encore très structurée dans le champ universitaire et académique. Qu'est-ce qui t'a amenée à aller vers cette discipline, quels détours, quelles rencontres, quelles lectures, quelle formation?

J'ai rencontré Pierre Bourdieu en Algérie en 1961 où j'avais rejoint mon compagnon. Je continuais ma licence de lettres modernes à l'Université d'Alger et découvrais concrètement la guerre de libération nationale aux causes de laquelle j'étais déjà acquise comme tous les progressistes. Bourdieu cherchait, par l'intermédiaire de Abdelmalek Sayad, des femmes pour enquêter dans les cités de la périphérie. Certaines l'avaient accompagné depuis « la métropole ». Moi j'étais sur place et cette proposition m'a immédiatement enchantée. A. Sayad avait constitué plusieurs équipes mixtes, un homme algérien soutenant le FLN et une femme française sympathisante. Je formais équipe avec un étudiant en médecine aussi ignorant que moi en sociologie. Nous partions sur le terrain, sans protocole, sans questionnaire, avec pour seule consigne d'interroger sous tous ses aspects la vie des femmes (moi) et celle des hommes (lui).

Je dirai que cette séquence en Algérie pendant la guerre de décolonisation m'a fait connaitre des situations où ma vie était en danger puisque condamnée par les étudiants appartenant à l'OAS à être traînée nue dans la rue Michelet (équivalente au boulevard Saint Michel) pour être une «alliée » du FLN ${ }^{1}$. En réalité, j'étais arrivée insouciante, avec un ciré noir (l'habit des CRS) et une écharpe de soie verte (couleur $\mathrm{du}$ drapeau algérien) et j'avais pris l'habitude de m'installer au restaurant universitaire du côté occupé par les étudiants arabes sans y prêter attention. Ma participation à l'enquête de Bourdieu n'a fait que consolider ma réputation de traître. Lorsque nous arrivions dans les cités nous étions accueillis par des jets de pierre lancés par les enfants insultant mon camarade en arabe « comment tu as épousé une Roumiya ... ». Mais comme j'ai été acceptée par les adultes, je passais des heures à interroger les femmes promptes à raconter leurs vies, y compris dans leurs aspects les plus intimes, apparemment heureuses de parler avec une française dans une langue mal maîtrisée. J'idéalise peut-être cet épisode mais dans mon souvenir il est à l'origine de mon goût pour la connaissance du monde social. Bourdieu m'a couverte d'éloges, j'étais une enquêtrice exceptionnelle et il m'a invitée à le rejoindre lorsque je rentrerais à Paris. Qu'a-t-il fait de ces enquêtes, nous n'en avons rien su. Chacun remettait son compte-rendu qui n'était jamais suivi d'échange par un premier dépouillement ni a fortiori par une analyse. Autrement dit je n'ai pas appris les bases du métier, j'ai seulement expérimenté les embûches d'un terrain au départ hostile et appris à construire la relation enquêteur/enquêté.

De retour à Paris je me suis inscrite en sociologie à la Sorbonne et j'ai sollicité Bourdieu qui m'a intégrée, comme enquêtrice, dans l'équipe réunie pour l'étude sur 
l'art moyen ${ }^{2}$. Je m'y suis trouvée isolée, privée de discussions et obligée de quémander une petite rémunération. Naïve, j'apprenais la division du travail intellectuel et m'en attristais. Je me suis éloignée de mon idole qui agissait aux antipodes de ses écrits et me suis limitée à le lire dans les années 1980-90, puis ensuite à suivre ses cours au Collège de France.

Tout en étant surveillante de collège, j'ai préparé une licence de sociologie (avec pour enseignants Gurvitch, Aron, Passeron) qui comprenait à l'époque un certificat d'économie politique, de psychologie sociale et d'anthropologie. Ensuite j'ai suivi des séminaires de l'EPRASS (Enseignement préparatoire à la recherche approfondie en sciences sociales) où les étudiants s'initiaient à la sociologie empirique ${ }^{3}$ ainsi qu'un cours de statistiques au CNAM. Passionnée par la connaissance du monde social je fréquentais les séminaires de Touraine qui m'avait remarquée parce que j'osais prendre la parole mais ne me proposera aucune aide pour entrer dans l'enseignement supérieur ou une institution de recherche. Ce fut Viviane Isambert-Jamati, qui créait alors une équipe de sociologie de l'éducation au CES (Centre d'études sociologiques), qui me proposa des vacations pour mener des travaux financés par la Délégation générale à la recherche ou le CORDES (institution d'une politique contractuelle en sciences sociales 1966-1975) dont ceux qui ont porté sur les inégalités géographiques de scolarisation. Elle m'incita à présenter ma candidature au concours d'entrée au CNRS en 1967 sur ce sujet, j'y fus admise ${ }^{4}$. Elle me laissa ensuite toute indépendance pour faire de la recherche à ma manière en tâtonnant à partir de mes lectures et de mes intérêts.

QUESTION : En 1972, tu publies dans la Revue française de sociologie, un article intitulé "L'État et l'école. L'école privée en France». Rétrospectivement, on ne t'imagine pas travaillant sur ce thème, d'autant qu'on te retrouve quelques années plus tard, en 1977, publiant un ouvrage sur les sidérurgistes lorrains chez Maspero (Le capital : Les travailleurs et l'école, l'exemple de la sidérurgie lorraine). Quels cheminements (intellectuels, institutionnels, pratiques, etc.) te font ainsi passer de l'école privée aux ouvriers ? Est-ce en fin de compte une continuité qu'on ne voit pas spontanément?

Ce n'est pas seulement les objets d'étude qui caractérisent le travail d'un-e sociologue, la conception qu'il a de celui-ci le définit tout autant. Moi j'ai toujours voulu lier la recherche à des perspectives de transformations sociales. En cela je n'ai fait que suivre l'avertissement de Durkheim qui disait de "vouloir étudier la réalité il ne s'ensuit pas que nous renoncions à l'améliorer ». Chacun de mes sujets d'étude est plus ou moins lié à ce type de préoccupation. Il en est ainsi de l'article intitulé L'État et l'école. L'école privée en France qui tire son origine du débat soulevé par la loi Debré de $1959^{5}$. Celle-ci instituait de nouveaux rapports entre l'État et les établissements privés au moyen de contrats qui ont autorisé la naissance, à côté d'un service public, laïque et obligatoire, d'une sorte de service privé d'enseignement. Certes pour avoir fréquenté une école catholique j'étais plus sensibilisée que d'autres à ce que l'on appelait encore «la querelle scolaire » et j'entrevoyais que s'amorçait un processus d'effritement du statut de l'école républicaine, mouvement qui se poursuit aujourd'hui sous d'autres formes et impulsé par d'autres acteurs ${ }^{6}$.

S'en tenir à la chronologie des publications peut induire des malentendus en faisant croire à une continuité là où il y a souvent discontinuité ou simultanéité. Publier un article demande moins de temps que d'écrire et faire éditer un ouvrage. Le capital, les travailleurs et l'école, l'exemple de la Lorraine sidérurgique que j'ai publié en $1976^{7}$, trouve son origine dans l'échec d'une étude antérieure que Viviane Isambert nous avait 
demandée (à Monique Segré et moi-même) et qui portait sur les variations géographiques de la scolarisation en France allant (pour les enfants de 11 à 14 ans

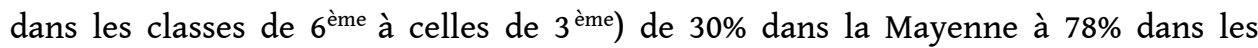
Basses-Pyrénées. Pensée en termes de mesure, utilisant des instruments statistiques inappropriés au phénomène étudié, aujourd'hui illisible, cette étude fut néanmoins publiée dans un numéro spécial de la Revue française de sociologie en 1968 dont l'objectif était de faire état, à parts égales, des travaux menés dans deux groupes de recherche: une équipe du CES (Centre d'études sociologiques) dirigée par Viviane Isambert-Jamati et le centre de sociologie européenne dirigé par Pierre Bourdieu'.

La stérilité de cette analyse m'a orientée vers une démarche toute différente, une sorte de monographie. La Moselle me paraissait un cas particulièrement intéressant car en 1968, 35,4 \% des enfants d'ouvriers âgés de seize à dix-huit ans étaient encore scolarisés à l'échelle nationale, alors qu'à la même date ce taux atteignait $60 \%$ en Ariège et seulement $28,2 \%$ en Moselle. Par ailleurs, l'édifice social tout entier portait la marque du grand capital avec les trusts de la sidérurgie. Plus de la moitié de la population active était constituée d'ouvriers de la grande industrie. Cette concentration de la classe ouvrière engendrait une forte polarisation des classes sociales. Dans ces années, les enfants nés et formés en Moselle étaient préparés à renouveler la force de travail fournie par leurs pères dans les usines et les mines. On y trouvait les principaux protagonistes qui se partageaient la formation des travailleurs : l'État, l'Église et le patronat à visage ouvert. Depuis ce terrain, j'ai vu advenir ce que je pressentais en raison de mon expérience: les CET (Collèges d'enseignement technique), méprisés par les uns mais recherchés par d'autres, sont un enjeu politique ${ }^{9}$.

Images 1 et 2 : Rue de la roquette, forge. Collège d'enseignement technique rue de la Roquette à Paris, 19..

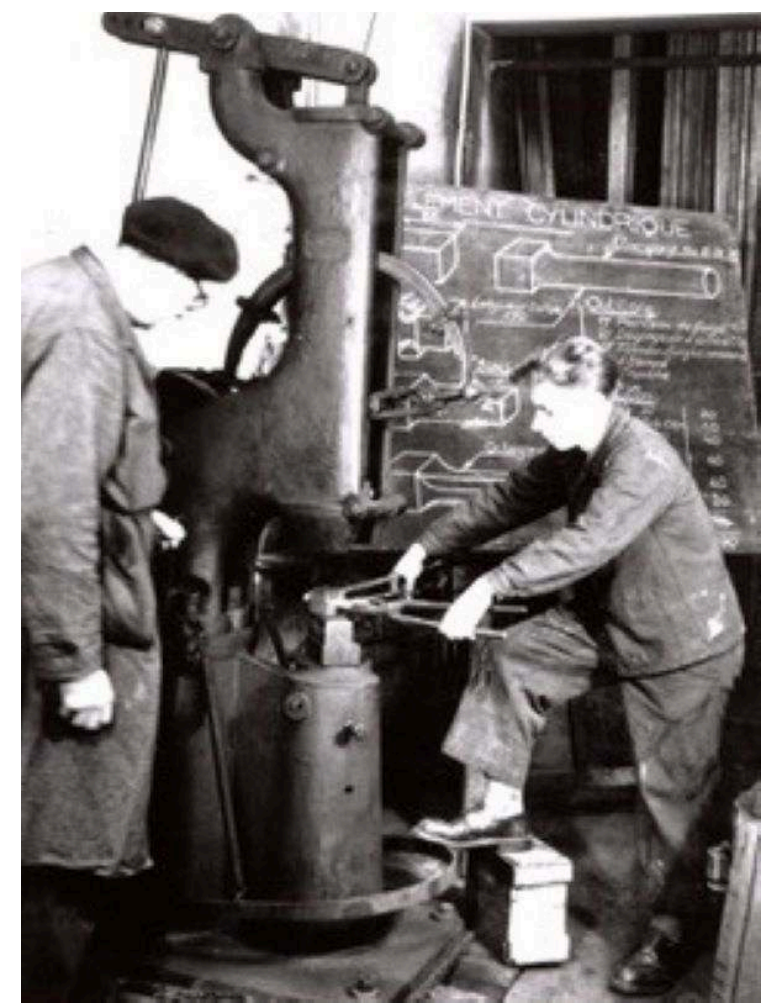


Image 2

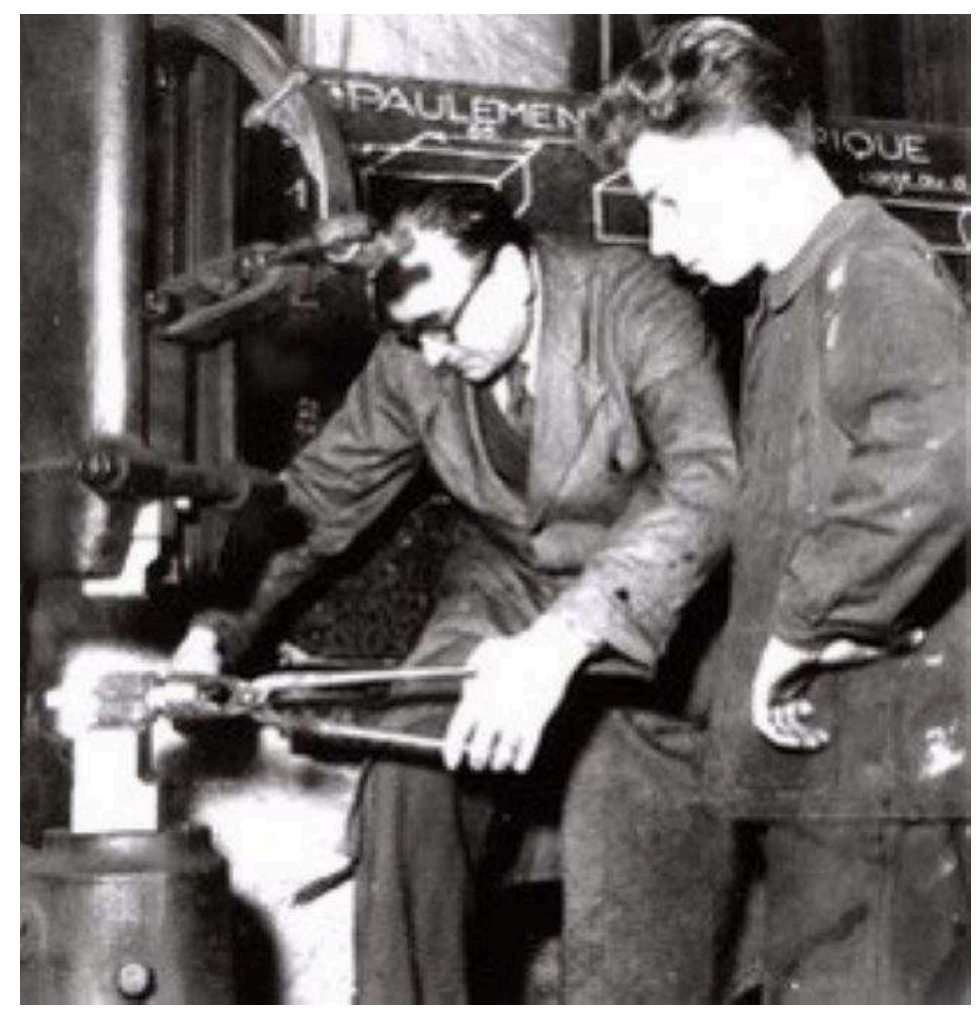

À noter l'importance du regard, celui de l'élève comme celui du maître (c)Daniel Tacaille ${ }^{10}$

Accessibles uniquement sur concours jusqu'en 1967 $7^{11}$, les CET délivraient des diplômes professionnels reconnus, jusqu'à ces deux dernières décennies, dans les grilles de classification des conventions collectives, reconnaissance mise en cause par la réforme du code du travail ; interprétation portée par les organisations ouvrières mais ignorée ou niée par des groupes politiques qui se prétendaient les portes paroles de la jeunesse collégienne dans les manifestations de l'époque «Les CET c'est déjà l'usine $»^{12}$. 

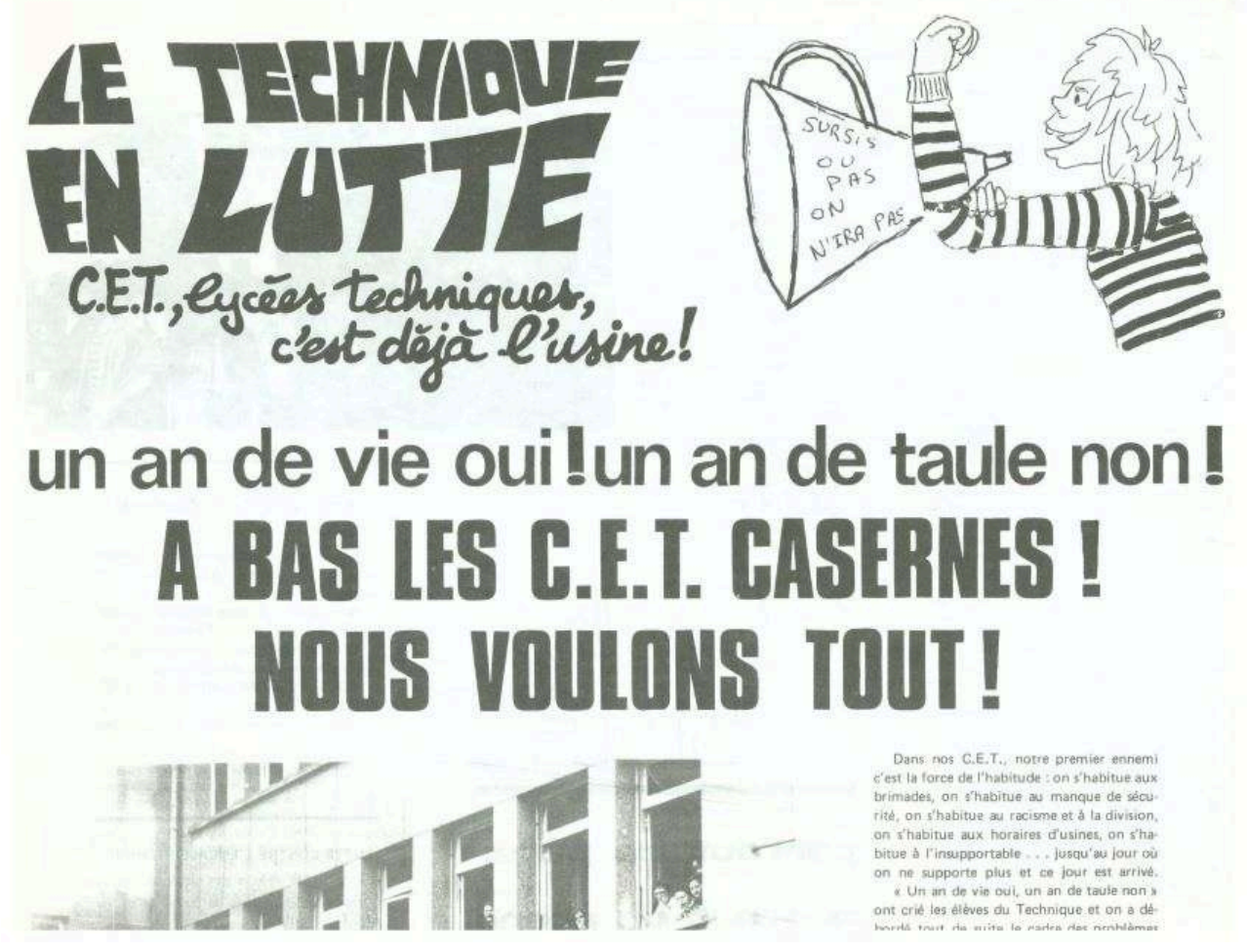

Archives personnelles de Lucie Tanguy

Je concluais cette étude sur une idée cultivée par le mouvement ouvrier depuis la Commune de Paris, l'Internationale de l'enseignement, les réflexions de Kroupskaia, de Maïakovski, de Gramsci : un enseignement polytechnique unissant le travail intellectuel et le travail manuel pour former des travailleurs cultivés aptes à organiser et diriger la production, à penser et agir sur tous les aspects de la vie sociale. Utopie dont l'esprit a besoin pour concevoir ne serait-ce qu'un horizon lointain.

Après cette recherche, je me suis sentie libérée de cette volonté de faire science sur le modèle des sciences de la nature, posture qui commandait encore mes travaux précédents comme l'article cité plus haut. Ce faisant j'embrassais la théorie marxiste ou plutôt une vulgate de celle-ci que je corrigerai par la suite.

QUESTION : Tu fais partie d'une génération marquée par la guerre d'Algérie, Mai 68 et les années 1970. Comment as-tu vécu cette période où les débats d'idées et les actions politiques étaient en pleine effervescence ? Quels engagements, quelles distances ? Quelles visions? Quelle place pour la sociologie dans ta compréhension de ce qui se jouait alors? Quels effets sur ta propre conception de la sociologie?

L'expérience de la guerre en Algérie, celle de la lutte des guerres de libération ("Vietnam-Laos-Cambodge même combat »), les manifestations antifascistes, et bien sûr l'explosion de mai 1968 avec tous ses extrêmes. Si j'ai participé à diverses actions allant de la distribution de pommes de terre aux ouvriers de Citroën, aux assemblées générales du CES durant lesquelles nous nous affrontions sur la conception des réformes ou révolutions à faire advenir. Je n'ai jamais cru que les conditions étaient réunies pour cela, je détestais les invectives de certains à l'égard de personnes plus âgées, membres du parti communiste, qui avaient risqué leur vie en s'engageant dans 
la résistance. Je manifestais comme tout le monde en criant « la recherche au service des travailleurs" sans adhérer aux slogans grandiloquents, parfois poétiques, revendiquant une liberté sans limites, "il est interdit d'interdire ». Je connaissais l'adversaire par expérience et savais qu'on ne peut sous-estimer sa force. J'admirais l'inventivité des étudiants qui avaient déclenché cette contestation radicale d'un régime politique et m'en méfiais parce que trop enfermée dans les idées.

Dans ces années-là, un courant tentait d'implanter un style de recherches réduisant les phénomènes sociaux à des variables, à la mesure des relations entre elles pour construire des modèles explicatifs. Le chef de file de ce courant de pensée était Raymond Boudon dont la thèse soutenue en 1964 avait pour titre L'analyse mathématique des faits sociaux dont R.Aron dit à l'auteur lors de sa soutenance « vous êtes l'incarnation vivante de l'accélération du progrès scientifique ». Sa publication fut reçue sur le même ton dans les revues académiques. La recension qui en est faite dans les Annales (en 1969) annonce « Voici un ouvrage dont on peut parier qu'il y sera fait référence dans un proche avenir comme "le Boudon" $»^{13}$. La méthodologie est à l'ordre du jour et R. Boudon avec P. Lazarsfeld (sociologue américain invité par lui) publieront des manuels réunissant un nombre important de recherches empiriques: Le vocabulaire des sciences sociales, concepts et indices (1965), L'analyse empirique de la causalité (1966) ${ }^{14}$. J'en ai gardé l'idée qu'une rigueur dans les méthodes d'observation et d'analyse est une exigence de toute connaissance objectivée, si possible scientifique et de celles de l'action. En 1968 je me suis éloignée de ce courant de pensée comme beaucoup d'autres de ma génération et, avec quelques amis engagés aux côtés des luttes des travailleurs, nous avons constitué un groupe de lecture du Capital où chacun devait, à son tour, animer la discussion sur quelques chapitres. Cette lecture appliquée, exigeante allait influencer notablement l'orientation de nos objets de recherche et de nos schémas d'analyse. Inconsciemment nous revenions à la théorie marxiste dont nos prédécesseurs s'écartaient (voir l'article de J.-D. Reynaud dans le premier numéro de la Revue française de sociologi $e^{15}$ ) après l'avoir embrassée quelques années auparavant.

La question de la séparation de l'école et du travail s'est exacerbée dans les années 1970 corrélativement à l'extension de la scolarisation. C'est dans ce contexte qu'émerge le principe d'alternance comme réponse aux contradictions inhérentes à un enseignement professionnel intégré dans l'école. La référence à l'expérience allemande, déjà célébrée par Jules Ferry et les ministres de l'Instruction publique qui l'ont suivi, revient comme modèle à expérimenter auquel s'opposent les partisans d'une forme de démocratisation scolaire. De fait, à la fin des années 1970, 40\% des jeunes Allemands âgés de 13 ans étaient orientés vers l'apprentissage. Réalisé pour l'essentiel sur les lieux de travail dans le cadre d'une cogestion entre le patronat et les syndicats de salariés, ce mode de formation s'accompagne d'une reconnaissance de l'ouvrier qualifié, passage obligé pour être promu contremaître, voire ingénieur de production. Cependant, ce mode de formation, amputé d'un accès à la culture générale, favorise-t-il une socialisation valorisant le travail source de connaissances et d'accomplissement de soi ? Telle était la question qui m'habitait dans cette enquête sur la formation professionnelle en RFA.

L'expérience de la RDA me paraissait tout aussi instructive. À partir d'une histoire commune avec la RFA, elle s'est construite, au lendemain de la seconde guerre mondiale, sur des bases économiques et politiques qui rompaient avec son passé : 
nationalisation des moyens de production et de distribution, planification de l'économie, direction de l'État se réclamant du marxisme-léninisme : le SED. En RDA, la liaison entre école et production constituait le principe de base de l'enseignement polytechnique caractéristique de l'école de 10 ans obligatoire et commune à tous. L'enseignement polytechnique, qui représentait le noyau d'une pensée ouvrière en matière d'éducation, s'est progressivement instauré dans ce pays au cours des années 1950-1980 ${ }^{16}$. Bonne occasion, par conséquent pour étudier l'école lorsqu'elle s'ouvre très tôt sur le monde de la production et du travail, pour observer comment et à quelles conditions le travail revêt une valeur éducative et peut devenir objet d'enseignement, bonne occasion aussi pour repérer à quelles conditions la production peut devenir une instance éducative (à côté et liée à l'école), pour analyser quels types de rapports ces deux instances entretiennent alors entre elles : de coopération, de domination ou de compromis?

Cette recherche sur la formation professionnelle dans les deux Allemagne s'est heurtée à une somme d'obstacles: une connaissance trop superficielle de ces questions dans chacun de ces pays, la difficulté de s'extraire de la représentation commune et, en RDA, le contrôle du parti dirigeant en situation d'enquête. L'ouvrage rendant compte de cette investigation, L'école et l'entreprise, l'expérience des deux Allemagne (avec Annick Kieffer, 1982 ${ }^{17}$ ) n'a guère suscité d'intérêt d'autant moins que la même année un autre paraissait signé de Maurice, Sellier, Sylvestre plus ambitieux: Politique de l'éducation et organisation industrielle en France et en Allemagne, essai d'analyse sociétale. Il s'y développait une toute autre analyse attribuant la puissance industrielle allemande à la politique éducative. Cet ouvrage devint vite une référence notamment parmi les sociologues du travail. L'interprétation qui y était avancée a été démentie cette dernière décennie par la croissance de la pauvreté, par la perte de centralité du « duales System ». Un numéro spécial de la revue Formation Emploi consacré à l'apprentissage en Allemagne, très documenté, fait voir toutes les transformations qui affectent le System dual depuis environ deux décennies. Loin de valider l'idéalisation qui en est faite par les politiques publiques et médiatiques françaises, le tableau dressé par les différents contributeurs fait ressortir les contradictions qui minent ce système et autorisent Mona Granato et Gilles Moreau à le qualifier de « colosse aux pieds d'argile $»^{18}$.

Un jour, je reçus une lettre de Lucien Sève me disant qu'ils (sous entendu les membres du parti communiste) n'avaient pas su s'approprier les idées de notre enquête sur la RDA. Notre ouvrage est sans doute tombé dans l'oubli faute, entre autres choses, d'intérêt pour ce type de questionnement et faute aussi de conclusions inspirantes. Nous y rappelions simplement que la liaison école-production a été de longue date revendiquée par une majorité du mouvement ouvrier et tout particulièrement par son courant marxiste qui voyait en elle un instrument permettant d'entamer la division sociale du travail, un moyen favorisant l'épanouissement de "l'homme» dans ses multiples capacités. Selon cette thèse, apprendre dans la production c'est apprendre à faire mais aussi à penser et organiser cette production et à décider de son sens. Or bien que se réclamant de la doctrine marxiste, l'école professionnelle en RDA s'attachait à former un travailleur averti et performant mais non un être social apte à penser le sens et l'organisation sociale de la production. 
Pour autant à vouloir défendre à tout prix une école séparée du monde du travail n'entretient-on pas le mépris du travail manuel et ne légitime-t-on pas son caractère dominé en niant sa valeur et sa nécessité, la hiérarchie des salaires et du positionnement social? Tels étaient les termes dans lesquels je pensais à cette époque, trop abstraits, enfermés dans une théorie marxiste trop doctrinale.

Durant la décennie 1980, j'approfondis la démarche mise en œuvre dans les études sur l'Allemagne, l'affinant sur le plan méthodologique, m'inspirant de la perspective avancée par des auteurs britanniques tels que Basil Bernstein et M.F.D. Young ${ }^{19}$; je tentais (avec Viviane Isambert-Jamati) d'infléchir le courant dominant en sociologie de l'éducation qui s'est moins intéressé à ce qui se passe dans cette "boîte noire " qu'est l'école qu'à ce qui en sort. Les sociologues anglais et américains se proposaient d'analyser le curriculum formel et caché, c'est-à-dire d'analyser la manière dont le savoir est organisé, transmis et évalué dans les institutions scolaires. Ces sociologues ont rempli leur programme en considérant le curriculum comme une sorte de relais de pouvoir qui leur sont extérieures (domination d'une classe sur d'autres, d'un sexe sur l'autre, d'une race etc.). À la différence de ces sociologues, dont les travaux n'ont été connus en France que dans les années 1980, je me suis appliquée à étudier concrètement les contenus d'enseignement, les caractéristiques sociales des enseignants et leurs pratiques.

Considérant que la production et l'appropriation des connaissances sont un aspect de la lutte des classes sociales je me suis proposé d'interroger l'institution éducative qui a pour mission première d'instruire le peuple dans sa totalité et sa diversité. Quelles catégories de savoirs l'école sélectionne-t-elle parmi l'ensemble des savoirs produits et en usage dans la société à un moment donné ? Comment organise-t-elle ces différentes catégories de savoir pour définir les programmes des diverses filières d'enseignement, en bref quels sont les mécanismes qui président à la distribution des savoirs ? Lors d'une conférence organisée par Viviane Isambert-Jamati à la Sorbonne M. Young s'est dit surpris par la construction des indicateurs utilisés pour répondre aux questions que je posais: la durée de la scolarisation, le temps attribué aux différentes disciplines, la valeur qui leur était accordée dans les examens etc. J'essayais, en effet, de combiner théorie et administration de la preuve par des indicateurs se prêtant à vérifier la véracité des faits observés (Savoirs et rapports sociaux dans l'enseignement secondaire en France, RFS, 1983) ${ }^{20}$.

Dans le même temps, je focalisais cette étude sur Les savoirs enseignés aux futurs ouvriers (Sociologie du travail, 1983) selon la même méthode montrant ainsi qu'à cette date, l'école assurait mieux l'apprentissage des techniques que celui des conditions de travail et c'est la discordance entre ces deux apprentissages qui justifiait, aux yeux des dirigeants, la recherche de nouveaux modes de formation basés sur l'alternance école-entreprise. Comparant des séries de sujets d'examens donnés aux épreuves de français du CAP et au baccalauréat on pouvait voir que l'enseignement professionnel distribue des connaissances sur la matière, sur les procédés de production mais non celles nécessaires à l'être social et au sujet politique (L'enseignement du français au LEP, miroir d'une perte d'identité (avec C. Agulhon et F. Ropé, Études de linguistique appliquée, 1982) 
Images $n^{\circ} 4$ et 5 : Atelier neuf à Bagnolet

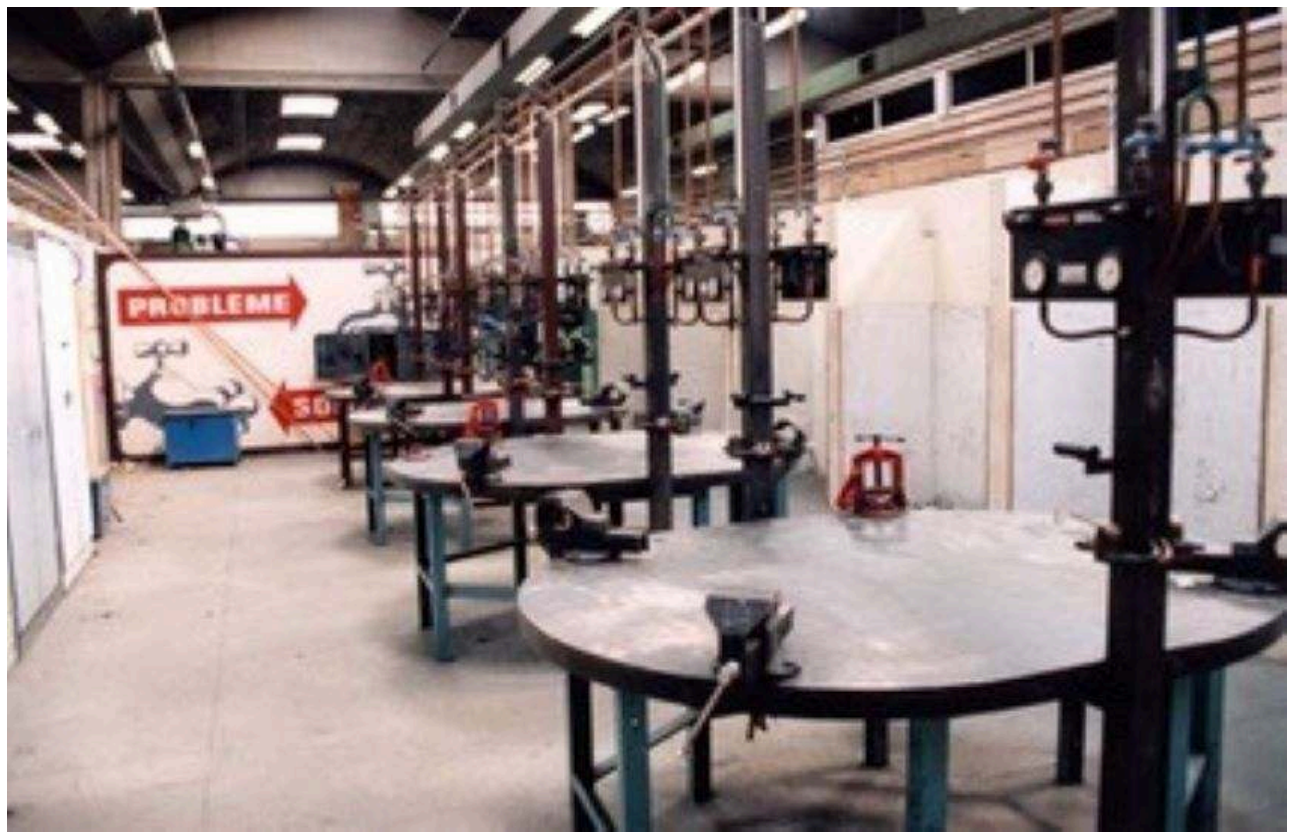

Image 5

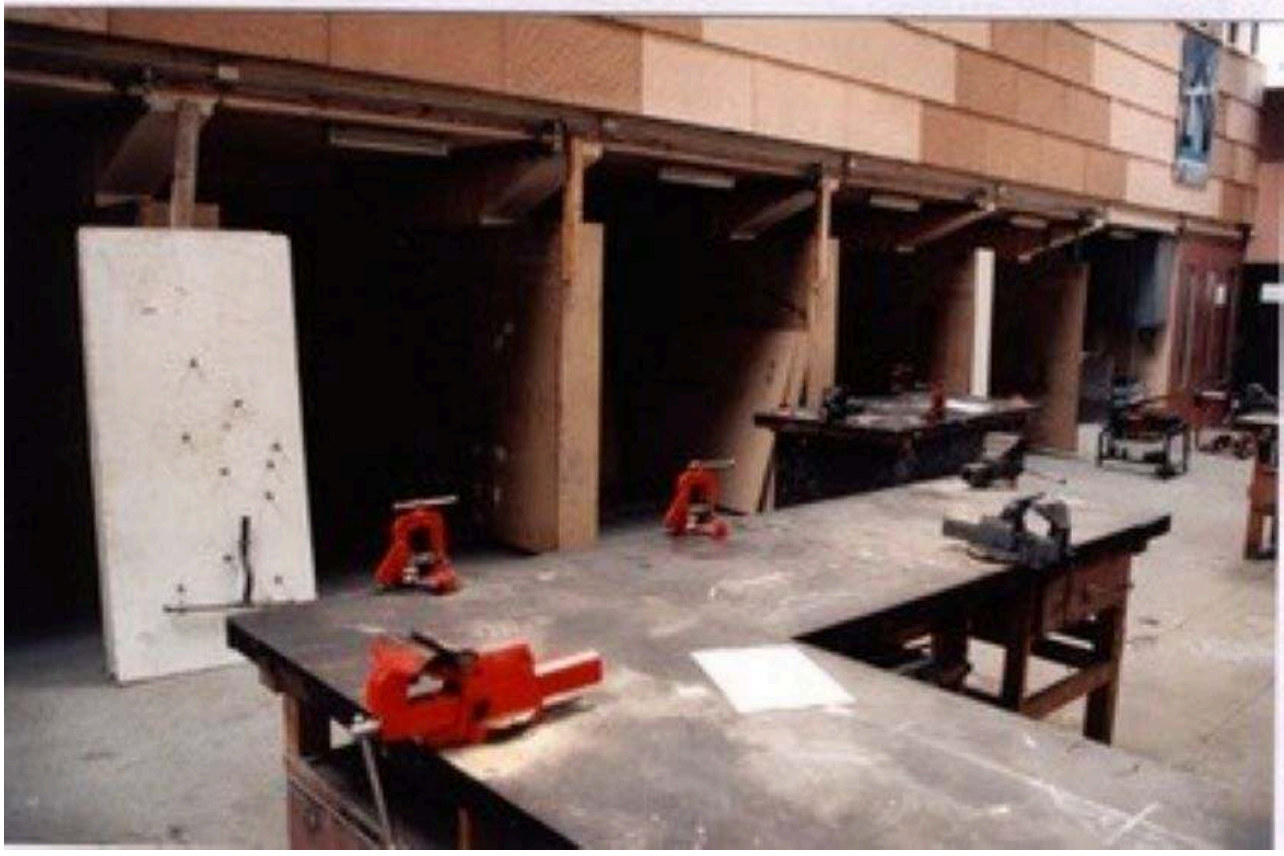

Les cabines permettent de réaliser une partie d'installation. C'est ce qui est généralement attendu pour le CAP. Dans certains établissements, les pièces sont conservées.

(c)Daniel Tacaille

À cette date, il me semblait avoir mené des recherches obéissant aux deux principes qui présidaient à mon engagement intellectuel : la division des savoirs distribués par l'enseignement général et l'enseignement professionnel constitutive de la division en classes sociales et l'objectivation des mécanismes en œuvre dans ce processus. Autrement dit, je restais attachée à une perspective inspirée du marxisme mais détachée de la théorie générale et soucieuse de restituer le plus rigoureusement 
possible mes observations et interprétations. Je ne crois pas que ces articles aient beaucoup intéressé les sociologues de l'éducation de par leur méconnaissance de l'enseignement professionnel, ni non plus les sociologues du travail qui n'ont pas retenu la formation initiale des travailleurs dans leur domaine d'étude. Un jour j'appris, par hasard, que cette étude sur l'enseignement du français dans les LEP était programmée dans les Instituts universitaires de formation des maîtres (IUFM) ${ }^{21}$.

La méconnaissance de la voie professionnelle qu'avaient les services administratifs du ministère de l'Éducation nationale fut l'occasion pour moi de poursuivre cette recherche sur les enseignants de l'enseignement professionnel. Une enquête extensive menée en collaboration avec le SPRESE (ensuite appelé DEP, Direction de l'Évaluation et de la Prospective) en 1983-84 sur un échantillon représentatif de la population d'enseignants en mécanique et en électronique, spécialités qui rassemblaient respectivement 34 et $16 \%$ des élèves scolarisés dans les LP industriels, a été suivie d'une enquête par entretiens auprès de 87 enseignants. Ces travaux ont été effectués en 1985-86, moment où les changements s'accéléraient dans les modes d'organisation de la formation et dans ceux du recrutement des enseignants qui marginalisaient ceux qui étaient issus de carrières ouvrières. Ces changements qui me sont apparus, en premier lieu, comme autant d'obstacles s'opposant au recueil d'informations standardisées se sont, au final, avérés être des conditions privilégiées pour observer le sens de ces changements, les modalités de leur mise en œuvre. La conscience très vive qu'en ont les agents, par les clivages et conflits qu'ils suscitent, leur inégal état d'avancement selon les établissements, offrent au sociologue des situations qui s'apparentent par maints aspects à celles vécues aujourd'hui comme le montrent les réflexions émises par un répondant sur cinq dans une rubrique «libres opinions » figurant en fin de questionnaire.

«Dans ces conditions nous allons vers notre mort? Qui nous croquera? Le privé? Arriverons-nous à la retraite? Peu probable. Avons-nous des têtes pensantes qui évitent de se succéder en innovant quelque réforme déjà dépassée ou inapplicable pour en récolter des fruits?"

(Professeur de mécanique, ancien OP, né en 1942, 9 ans d'ancienneté à l'EN).

"S'il me restait encore une once d'espoir que pourrait réveiller l'objet de cette enquête mes vœux seraient pour une réelle adaptation de nos établissements aux réalités d'un monde en continuelle évolution technologique (équipements de nos établissements et formation des enseignants.)"

"On nous demande beaucoup, on nous donne RIEN".

"On arrive même au paradoxe extrême de former des techniciens de l'industrie dans des

domaines où nous n'avons même pas été recyclés ${ }^{22}$. "

(Ancien OP électronicien, 46 ans, 20 ans d'ancienneté à l'EN).

Il suffit alors d'associer les règles du travail scientifique aux qualités d'observateur et d'avoir une bonne écoute de l'autre pour capter la diversité d'une réalité en mouvement. La démarche sociologique rejoint avec profit celle de l'ethnologie (observations du milieu dans son ensemble, des ateliers, participation au repas, aux pauses-cafés, etc..).

Le changement en cours qu'était le recrutement de diplômés de l'enseignement technique supérieur au lieu et place de celui d'une élite d'ouvriers professionnels institué dans les années 1940-60 transformait inévitablement les pratiques pédagogiques et les contenus enseignés. Les savoirs de métiers font place aux savoirs techniques. L'analyse développée à partir de ces enquêtes et observations in situ qui donna lieu à un ouvrage L'enseignement professionnel en France, des ouvriers aux 
techniciens (1991) ${ }^{23}$, obéissait à un double dessein : faire œuvre de mémoire en consignant les pratiques et les valeurs de ces anciens ouvriers professionnels qui ont bâti cet enseignement pendant plus de trente ans ; construire une méthode d'analyse qui éclaire la nature des institutions par ce que sont ou deviennent leurs agents et qui s'applique aux formes et contenus de cette activité sociale, la formation professionnelle.

À l'image du statut des personnes enquêtées, l'étude sociologique de cette catégorie d'enseignants resta plutôt dans l'ombre au regard de celle à laquelle elle s'opposait peut-être trop timidement. C. Grignon voyait dans l'enseignement professionnel un apprentissage de la discipline et de la soumission alors que j'entendais montrer qu'il transmettait les savoirs et savoir-faire constitutifs de la qualification des ouvriers et ouvrières reconnue sur le marché du travail ${ }^{24}$. C'est aux côtés de ces gens de métier que j'ai compris l'enjeu que représentait la formation professionnelle pour la condition ouvrière. En passant un long temps sur le terrain à les observer, en analysant leurs pratiques, leurs visions des changements en cours, sans chercher à théoriser trop rapidement, j'entrevoyais que cet enseignement s'orientait vers le profil du technicien, délaissant celui de l'ouvrier qualifié. L'ensemble de ces recherches accomplies dans les années 1980, me conférait une spécialisation peu attractive : l'enseignement professionnel. Rien d'étonnant donc qu'à la fin de cette décennie, je fus sollicitée par le secrétariat d'État à l'Enseignement technique et professionnel pour réaliser une mission destinée à légitimer le « niveau $\mathrm{V}$ » nouveau nom donné à l'enseignement professionnel, en référence à la nomenclature des niveaux de formation élaborée durant les $\mathrm{IV}^{\mathrm{e}}$ et $\mathrm{V}^{\mathrm{e}}$ Plans $^{25}$. Je reviendrai plus loin sur cette expérience.

QUESTION : Plusieurs de tes travaux ont une dimension socio-historique marquée, à l'instar de l'ouvrage Les instituts du travail. La formation syndicale à l'université de 1955 à nos jours (2006) ou de La sociologie du travail en France. Enquête sur le travail des sociologues (1950-1990) (2011). Quelle est ta conception des rapports entre histoire et sociologie? Quelles ont été tes collaborations de recherche avec les historiens et en quoi y vois-tu des vertus heuristiques? En quoi l'histoire permet de réfléchir aux autres chemins qui auraient été possibles?

Je crois pouvoir dire que la quasi-totalité de mes travaux, à l'exception des tous premiers, s'inscrivent dans une perspective socio-historique. A priori, ce domaine de recherches sur les enseignements professionnels et techniques ne s'y prêtait pourtant pas : ancrés dans le présent et constamment sommés de se projeter dans le futur, pour anticiper les qualifications projetées nécessaires par les représentants des grandes entreprises dans les années à venir, ils appellent plutôt des études arrimées au débat social. Mais n'oublions pas que c'est en se dégageant des conflits les plus visibles que les chercheurs en sciences sociales sont parvenus à définir des questionnements et des schémas d'intelligibilité d'une réalité façonnée par ces conflits et les compromis de leur résolution. Les historiens ont devancé les sociologues sur ce chantier.

Au cours de la décennie 1980, les enseignements professionnels et techniques ont fait l'objet de réformes répétées, pour la plupart en prolongement de celles impulsées durant les décennies précédentes, et qui concernent aussi bien les contenus d'enseignement (redéfinis à partir de référentiels d'emplois), que les diplômes (la création du baccalauréat professionnel par exemple), les modes de recrutement des enseignants, l'instauration de toutes sortes de stages en entreprise, etc. Ces 
interventions politiques, souvent élaborées en réponse aux évolutions sociales et démographiques - et au nom de la valorisation de ces enseignements - se disent vouloir les mettre à parité avec l'enseignement général, qui reste le modèle de référence. C'est dans ce contexte que des historiens et des sociologues vont se rencontrer après avoir cheminé dans leurs disciplines respectives, et former un petit cercle d'échanges et de coopération. Le numéro spécial de la revue Formation Emploi (1989) peut être considéré comme une illustration de cette rencontre d'intérêts d'historiens et de sociologues pour explorer un chantier que Pierre Caspard, directeur du Service d'histoire de l'éducation à l'INRP, estimait encore déserté à cette date ${ }^{26}$. La note de synthèse que je fus invitée à réaliser quelques années plus tard n'est pas exempte de subjectivité puisque faisant moi-même partie de ce petit milieu de recherche ${ }^{27}$.

Cette façon de faire "avec l'histoire », qui n'avait pas été préméditée, s'est imposée comme une évidence provenant tant par l'objet lui-même que par mon apprentissage en sociologie aux côtés de Viviane Isambert-Jamati qui développait une perspective socio-historique au moment où le structuralisme triomphait (Crises de la société, crises de l'enseignement $(1970)^{28}$, Les savoirs scolaires, enjeux sociaux des contenus d'enseignement et de leurs réformes (1990)). Elle le faisait sans s'en réclamer à voix haute, à la manière durkheimienne de L'évolution pédagogique en France (1938) en soulignant la pluralité des temps sociaux. Plus que l'enseignement général, les formations techniques et professionnelles ont toujours été objets de critiques parce qu'inadaptées aux impératifs techniques et économiques du moment. Comme beaucoup de jeunes chercheurs j'avais tendance à voir du nouveau là où seules les formes d'un même phénomène avaient changé. Je maugréais parfois mais j'écoutais celle qui m'apprenait à penser le social en mouvement. Plus tard, j'ai travaillé plusieurs années avec Guy Brucy (historien) qui m'entrainait à observer les différentes temporalités de l'évolution des institutions, des contenus d'enseignement, des enseignants. Sociologue de formation après une agrégation en mathématiques, J.-M. Chapoulie a lui aussi toujours plaidé pour observer les questions sociologiques dans la durée afin d'éviter les effets pervers du présentisme.

Instrument de socialisation à la hiérarchie sociale, l'école est aussi un enjeu politique pour accéder à la connaissance, aujourd'hui mise en cause par la célébration des vertus de l'apprentissage en entreprise et l'acquisition de l'esprit entrepreunarial. Pour mieux apprécier l'ampleur, le sens des changements en cours, j'ai rappelé à grands traits des projets et des expériences qui ont fait date et qui ont constitué des sources de propositions. Ce retour sur le passé vieux de plus d'un demi siècle ne procède pas d'une quelconque nostalgie ni encore moins d'une croyance en la possibilité de le faire renaître. Des historiens comme Marc Bloch ont rappelé avec insistance que l'ignorance du passé est préjudiciable non seulement à la connaissance du présent mais à l'action elle-même. Telle est la conviction qui m'anime quand je reviens vers certaines périodes de l'histoire où des expériences d'émancipation ont pu s'accomplir. Les extraire de l'oubli est une des tâches du sociologue et de l'historien afin de faire entrevoir des possibles à faire advenir. Ainsi le célèbre plan Langevin-Wallon a énoncé, au lendemain de la seconde guerre mondiale, les bases d'une refonte du système éducatif. Bien que resté sans application, ce texte a constitué une référence souvent invoquée pour justifier les réformes scolaires jusqu'aux années 1970. Il représente encore l'expression d'une école démocratique, 
dépassant les limites de l'école républicaine ${ }^{29}$. Pour ces réformateurs, il s'agissait de former à la fois des techniciens compétents dans leur domaine et des citoyens éclairés. Ils ne poursuivaient pas l'instauration d'une société sans inégalités mais une société où les élites seraient multiples. Pour eux, l'acte éducatif est un acte politique, et la pédagogie n'est pas seulement l'affaire des enseignants, car l'égalité des droits à l'éducation est aussi un droit des travailleurs à l'émancipation intellectuelle et à la culture. Georges Friedmann, un des fondateurs de la sociologie du travail en France, est aussi une figure de ce courant de pensée attaché à définir un enseignement technique qui rivaliserait avec l'enseignement secondaire scientifique et littéraire. Il s'est efforcé de convaincre ses contemporains que «l'humanisation de la technique est une des grandes missions et sans doute même l'essentielle du XX $\mathrm{XX}^{\mathrm{e}}$ siècle. C'est en première urgence dans la formation des citoyens qu'elle doit être pensée, cherchée, réalisée $»^{30}$. Cette idée de mettre en débat les fondements de l'universalisme associé à l'enseignement général me paraît toujours à cultiver aujourd'hui et peut-être plus qu'hier.

Trouvant nécessaire de revenir sur ma discipline qui s'était si peu intéressée au domaine d'investigation qui est devenu le mien, je me suis décidée à interroger à nouveaux frais l'histoire de la sociologie en France. L'enquête que j'ai entreprise sur la naissance de la sociologie du travail en France et son déploiement jusqu'aux années $1990^{31}$ était un nouveau projet qui s'imposait après l'enquête portant sur les Instituts du travail ${ }^{32}$. Selon la majorité des auteurs qui se sont intéressés à l'histoire de la sociologie du travail en France et les manuels qui la diffusaient, celle-ci serait née au Centre d'Études sociologiques (CES-CNRS). À l'examen de faits restés ignorés et découverts dans les archives, il m'apparaissait que cette proposition devait être corrigée pour faire ressortir la place occupée par l'Institut des sciences sociales du travail dans la naissance de cette discipline. Créé à l'initiative du ministère du Travail, cet institut se voit rapidement assigner une mission de recherche appliquée aux problèmes $\mathrm{du}$ travail. Cette politique, impulsée dans le cadre de la «modernisation » de la France dans les années 1950, rencontre les attentes de jeunes chercheurs recrutés depuis peu au CNRS. L'ouvrage cité plus haut relate la concrétisation de cette rencontre et l'héritage qu'elle a laissé : un style, un courant de recherche et une revue.

Cette recherche réalisée essentiellement à partir de diverses sources d'archives combinées à des entretiens s'est avérée, à mes yeux, particulièrement féconde au terme d'errements dans son déroulement. L'apprentissage du métier de sociologue place au cœur des cursus y conduisant l'enquête par questionnaires ou par entretiens, l'observation des situations, l'exploitation de sources secondaires à caractère statistique notamment, mais pas à la consultation et au dépouillement d'archives. C'est pourtant cela que Fernand Braudel préconisait dans sa dispute avec Georges Gurvitch (1958) sur la prise en compte du temps dans les sciences sociales en déplorant l'ignorance dans laquelle les sociologues la tiennent. Aux yeux de cet historien, en s'en tenant à observer l'actuel, la sociologie se cantonne à être " une sociologie événementielle qui encombre nos bibliothèques et les cartons des gouvernements et des entreprises, qui est sans valeur scientifique parce qu'ainsi devenue incapable d'enregistrer et de comprendre le sens du mouvement de tout phénomène social ». Et d'ajouter, pour mettre fin aux conflits entre historiens et sociologues, son souhait de voir les jeunes sociologues prendre «sur leurs années 
d'apprentissage, le temps nécessaire pour étudier, même dans le plus modeste des dépôts d'archives, la plus simple des questions d'histoire, qu'ils aient une fois au moins hors des manuels stérilisants, un contact avec un métier simple, mais que l'on ne peut comprendre qu'à le pratiquer, comme tous les métiers sans doute ${ }^{33}$ ».

J'ai découvert, assez tard, la justesse de ce jugement, après avoir fouillé dans les dépôts d'archives aussi divers que la Maison des mineurs de Piennes (pour étudier la reconversion industrielle du pays minier de Lorraine), celles de l'association des premiers directeurs du personnel de grandes entreprises (pour voir comment la formation professionnelle continue a été promue dans celles-ci), celles du Plan (pour rappeler comment la notion de formation a été promue au lieu et place de celle d'éducation et mise en rapport avec l'emploi) ou encore celle des Instituts du travail mentionnés plus haut et du CNRS. Le travail sur archives est, à mes yeux, un gardefou contre les formalisations précipitées et les théories abstraites, ou simplement contre « les discours sur ». Ainsi ai-je avancé à petits pas, par essais et erreurs pour rendre plus intelligibles les diverses questions sociales que j'ai abordées.

QUESTION: Tu es reconnue en sociologie, entre autres, pour ta volonté systématique d'articuler sociologie du travail et sociologie de l'éducation, et donc de décloisonner un champ académique constitué de sociologies spécialisées qui sont peut-être autant de « potentat» ou de «chasse gardée ». Comment s'est construit chez toi, intellectuellement, cet impératif de lier sociologie de l'éducation et sociologie du travail ? Quelles finalités? Quelles vertus heuristiques y vois-tu ? En quoi ton message a-t-il été entendu?

Comment répondre à ces questions autrement qu'en admettant des liens où circonstances et hasard ont sans doute tracé le chemin. Après coup, une fois le chemin parcouru, je pourrais dire parce que le travail a été premier par nécessité au début de ma vie et que l'école apparaissait, aux yeux de ma mère, comme la voie qui allait permettre à ses filles d'accéder à une meilleure condition sociale qu'elle, tout en restant près de la famille et de travailler au pays. Mais n'est-ce pas là une interprétation rationnelle qui ne résiste pas à une quelconque analyse objectivée de ma subjectivité. Alors dois-je évoquer mon entrée au CNRS tenue en main par Viviane Isambert-Jamati qui, elle, passait de la sociologie du travail à la sociologie de l'éducation? Sans doute mais c'est insuffisant. Si je laisse mon esprit réveiller ce lointain passé, apparaît Pierre Naville, un des fondateurs de la sociologie du travail qui, à ses débuts en 1959 , déclare ${ }^{34}$ « C'est par le biais de la démographie, de l'économie politique et des préoccupations de l'orientation professionnelle, jointes à une intervention toujours plus étendue de l'État dans la vie publique et privée que les réalités scolaires commencèrent à entrer dans le champ de l'enquête sociale et systématique ". J'emboitai ses pas dès mon entrée au CNRS où il était Directeur de recherche et lui demandai de diriger ma thèse qui donna lieu à l'ouvrage sur la Lorraine. M'attribuant, lors de nos premières rencontres, toutes les qualités d'une chercheuse potentiellement créative, me laissant faire mon chemin jusqu'à la soutenance où, au vu des résultats, il se déclara déçu. Faut-il également rappeler que dans les années 1970, la spécialisation en sciences sociales n'était pas aussi accusée qu'aujourd'hui?

Avoir tenté de reconstituer les débuts de la sociologie du travail et son évolution jusqu'aux années 1990 et non ceux de la sociologie de l'éducation trouve, pour une part, sa raison d'être dans mon appartenance, à cette date, à un laboratoire de sociologie du travail mais aussi au statut de ces deux branches de la sociologie. La sociologie du travail, basée sur des enquêtes de terrain fournissait plus de références, 
de manuels, d'investigations empiriques et d'archives. Par ailleurs, la sociologie de l'éducation, plus récente, marquée par l'empreinte des figures tutélaires de Pierre Bourdieu, de Jean-Claude Passeron et de leurs disciples, s'adossait progressivement aux sciences de l'éducation créées dans l'Université par le décret de $1967^{35}$. Ces différentes raisons ont pu me conduire vers ce domaine de questions sans m'intégrer dans l'une ou l'autre spécialité. Ce faisant, je restais à la frontière de ce que Bourdieu a appelé des champs, position inconfortable pour déployer des recherches mais indispensable pour montrer les relations qu'ils entretiennent entre eux.

Les transformations sociales qui s'opéraient simultanément dans les années 1980-90 sous le terme de compétences, dans différentes sphères d'activités telles que l'économie, le travail, l'éducation et la formation, nous offraient une nouvelle occasion d'observer et d'analyser leurs relations. Fortement liée à l'idée de performance et d'efficacité dans chacun de ces domaines, la notion de compétences tend alors à se substituer à d'autres qui prévalaient antérieurement, comme celle de savoirs et de connaissances dans la sphère éducative, ou celle de qualification dans le travail. Celles-ci ne disparaissent pas pour autant mais elles perdent leur position centrale et, associées à celles de compétences, elles suggèrent d'autres connotations. En bref, la notion de compétence devenait une notion témoin de notre époque. F. Ropé et moi-même avons cherché à identifier les changements, à caractériser leurs orientations, à faire ressortir les homologies qu'ils présentent ${ }^{36}$. Pour cela nous avons réuni des chercheurs appartenant à des disciplines différentes allant de la sociologie à la psychologie cognitive en passant par la didactique, chacun s'emparant de la question pour la traiter sur des modes spécifiques. Ainsi avons-nous tenté d'analyser l'analogie existant entre les techniques, les catégorisations mises en œuvre dans les entreprises pour identifier et évaluer les compétences des travailleurs en exercice et celles utilisées dans l'institution scolaire pour définir les savoirs à transmettre aux élèves et ensuite évaluer leur acquisition. Certes, nous n'avons pas manqué de le montrer, ces homologies sont le résultat de processus, de chaînes de relations très différentes mais il nous paraît primordial de ne pas perdre de vue le «parallélisme des transformations sociales » car l'une des «tâches » des sciences sociales consiste à mettre en évidence "l'orientation commune à toutes les transformations des relations humaines, non seulement dans une sphère particulière mais dans toutes les sphères » (Norbert Elias, 1970, 1986 ${ }^{37}$ ). L'émergence de notions communes, comme celle de compétences, témoignent de changements transversaux à une société et de leur interdépendance.

La conception et la réception d'un ouvrage collectif que j'avais dirigé dans les années 80, L'introuvable relation formation-emploi (1986) m'avait déjà fait éprouver le besoin impérieux pour les chercheur-es en sciences sociales, de réfléchir aux outils avec lesquels ils pensent, d'examiner les mots, les notions qu'ils utilisent, tant ceux-ci participent de la constitution de la réalité sociale. A posteriori, je me suis rendue compte que, par le type d'analyse qu'il faisait d'un questionnement social, cet ouvrage avait contribué à donner forme à des représentations, à des politiques qui cherchaient à établir des correspondances entre deux ordres de phénomènes séparés, la formation et l'emploi, et à faire apparaître ces relations comme nécessaires alors que son propos était à l'opposé. En bref, par maints aspects, cet ouvrage confortait une propension à parler de "la relation formation-emploi » au singulier et à substantifier des phénomènes qui ne peuvent être pensés qu'en termes de processus. 
La majorité des études menées aujourd'hui dans ce domaine font apparaître la formation comme la condition première d'accès à l'emploi en occultant les rapports sociaux qui président à cette relation entre diplômes et places dans la hiérarchie sociale. À l'exception des différences de sexe qui sont prises en compte - peut-être parce qu'elles se prêtent facilement à la mesure, mais surtout parce que le mouvement des femmes a fait reconnaître ces différences comme fait social - les inégalités sociales paraissent aujourd'hui résulter des inégalités scolaires.

Cet ouvrage a été très diffusé et, à mon grand dam, je fus sollicitée dans différents lieux sur la base d'une méprise totale sur son contenu, méprise que facilitait son titre qui pouvait faire entendre cette relation comme nécessaire et non comme contenant divers possibles.

QUESTION : Toujours en exergue du livre Enseigner l'esprit d'entreprise à l'école (2016), tu donnes une définition de la sociologie, en y écrivant qu'en tant que sociologue «[tu t']emploie[s] à chasser les mythes qui nous aliènent ». On peut y voir implicitement une référence à la déconstruction des «prénotions » chère à Émile Durkheim, mais aussi la revendication d'une sociologie émancipatrice. Bref, à quoi sert la sociologie selon toi ? Quelle a été la réception de tes travaux? Que penses-tu de l'usage que font parfois les pouvoirs publics des travaux et recherches des sociologues?

La réponse à une question aussi vaste qu'il y a de sociologues ne peut être que plurielle. J'ai embrassé la sociologie comme une science qui, par les connaissances qu'elle produit, pouvait contribuer à orienter les transformations sociales vers plus de justice, d'égalité, de solidarité, soit vers les valeurs qui m'animaient dans la vie. Au fil du temps j'ai dû abandonner cette ambition et me rabattre sur des objectifs plus accessibles: comprendre le monde dans sa diversité, produire des connaissances issues d'enquêtes sur le terrain qui soient vérifiables. Je le répète pour moi, les sciences sociales doivent montrer qu'il n'y a pas d'évolution nécessaire et que toute situation qui fait problème contient en elle plusieurs réponses possibles qui ont des probabilités inégales de s'actualiser.

Une mission confiée par le secrétaire d'État à l'enseignement technique et professionnel (en 1990) a été, pour moi, l'occasion de mettre en œuvre une démarche qui invalidait l'idée qu'une politique d'élévation du niveau de formation était nécessaire. Cet organe de l'État me demandait de l'aider à résoudre la désaffection de l'enseignement professionnel, tout en légitimant les grandes orientations de la politique scolaire du ministère de l'Éducation nationale. Tout me portait à souscrire aux raisons invoquées pour rendre compte de cette situation : l'évolution du travail, le déclin du travail ouvrier, la perte du pouvoir d'identification de l'ouvrier qualifié au profit de l'extension du travail technicien avec notamment l'avènement de la figure du technicien d'atelier. En reconstituant la chaîne des relations qui va de la demande singulière de certaines catégories d'entreprises en compétences professionnelles, à l'élaboration d'une demande sociale générale, puis à sa traduction en termes de savoirs et savoir-faire à transmettre, et enfin à la définition de diplômes y correspondant, il devenait possible de montrer qu'une croyance collective apparemment partagée (en l'élévation du niveau de formation) occultait des désaccords profonds et que d'autres évolutions possibles étaient contenues dans cette situation. Ces désaccords laissaient d'ailleurs entrevoir les effets pervers d'une politique qui, en élevant la norme au niveau du baccalauréat, accentuait les classements opérés par l'école en marginalisant les jeunes qui n'y souscrivaient pas et généraient les contradictions aujourd'hui vécues par les bacheliers qui se sont 
identifiés à la figure du technicien pendant leurs études et qui se trouvent ensuite en majorité à des places d'ouvriers. En élargissant l'investigation et en prenant en compte les divergences et les contradictions entre les discours et les faits, il devenait possible de montrer que le recours exacerbé à l'école pour résoudre les problèmes du travail, et plus généralement de l'emploi, ne relevait pas d'une nécessité mais d'un ensemble de décisions, de choix opérés par telles ou telles catégories d'acteurs dans une conjoncture historique donnée. Il devenait également possible de reconstituer la chaîne des actions collectives et individuelles (ou tout au moins ses principaux maillons) pour montrer comment celles-ci avaient privilégié cette orientation au détriment d'autres possibles.

Cette mission a donc été, pour moi, l'occasion de revenir sur la tâche du sociologue, y compris et, je dirai surtout, dans une situation d'expertise publique. Comme le souligne $\mathrm{N}$. Elias «C'est en objectivant sa propre position que le chercheur peut instituer une distance par rapport aux dépendances qui le contraignent sans qu'il le sache ... (et qu'il parvient) à séparer le savoir "scientifique» des représentations immédiates, des préjugés spontanés ${ }^{38}$. Si tout le monde s'accorde pour dire que cette tâche consiste à rendre compte du réel, il n'y a pas nécessairement d'accord pour dire que le réel ne se confond pas avec les formes objectivement actualisées. Car le réel comprend également le non-actuel, les possibles qui ont été écartées. Si bien que la compréhension des faits actualisés suppose de les confronter aux possibles contenus dans les situations observées. Ainsi une sociologie critique, que je revendique, est une sociologie des possibles et non une sociologie de la nécessité.

Ce rapport d'expertise n'a eu qu'une influence limitée sur la politique scolaire puisque la réponse apportée au problème soulevé par la lettre de mission s'est réduite à restaurer un diplôme (le $\mathrm{CAP}$ ) qui certifiait une qualification ouvrière et que l'école a invalidé. Nos analyses n'auraient guère été diffusées si elles n'avaient pas rencontré d'autres attentes et d'autres intérêts. En faisant ressortir les contradictions générées par une politique "d'élévation du niveau », de substitution de compétences techniques à des compétences professionnelles dans un système productif considéré à tort comme relativement homogène, ce rapport a été très débattu et a fait débattre. Il a été, pour les mêmes raisons, écarté par les uns et utilisé par les autres, soit essentiellement par des milieux professionnels (syndicats d'enseignants de lycées professionnels, syndicats de salariés, organismes de formation des maitres de la formation continue, organisations et associations professionnelles patronales, organismes d'insertion professionnelle des jeunes etc.). En somme, l'accueil fait à cette expertise exprimait le bien-fondé du point de vue que j'avais adopté au départ : reformuler la demande qui m'était faite, comprendre les problèmes sociaux qu'elle occultait, éclairer les choix possibles contenus dans la situation du moment en spécifiant leurs sens et leurs effets, en bref instruire les acteurs concernés avant d'aider à la décision et de guider les politiques.

Cet exercice m'a fait prendre conscience de la profondeur du fossé qui sépare le monde du travail du monde scolaire, déjà déplorée par les réformateurs d'aprèsguerre. Pour les représentants des salariés, ce rapport était une reconnaissance de leur travail et de leur dignité. La majorité des agents de la haute administration de l'Éducation nationale (recteurs d'académie, inspecteurs) et des enseignants du secondaire ont, au contraire, violemment rejeté l'analyse faite et ses conclusions. Pour eux je "parlais comme les patrons", je m'opposais «à ce que les ouvriers 
deviennent des bacheliers ", voire encore "j'allais débarrasser les universités de ces bacheliers crasseux ». Le Secrétaire d'État lui-même a annulé, du jour au lendemain la conférence de presse prévue en me faisant savoir que ce rapport était " hors sujet » pour n'avoir pas répondu à la question qui m'était posée.

Par la suite, je revins sur la manière dont j'avais accompli cette mission officielle afin de mettre en perspective l'activité d'expertise avec celle de la recherche académique et, plus généralement, de reconnaitre les différentes formes de production des connaissances sur le monde social ${ }^{39}$.

Autrement dit, un chercheur peut accompagner à son insu une politique dont il n'avait pas déchiffré le sens (faire de la formation un instrument assurant l'équilibre de l'emploi) ou à, l'inverse, montrer que les changements impulsés accentuent les inégalités sociales. Lors de cette mission il me fut conseillé d'éviter le terme "ouvrier» déprécié par les élèves et les enseignants eux-mêmes. En réponse, j'intitulai le rapport publié à la Documentation française Quelle formation pour les ouvriers et les employés en France ?40

QUESTION : Dans un numéro de 1989 de Formation Emploi auquel tu as contribué, Pierre Caspard lançait en postface un cri d'alarme, désormais célèbre, face au «chantier déserté ${ }^{41}$ » de l'enseignement technique et professionnel. Onze ans plus tard, dans un état des lieux que tu publiais dans la Revue française de pédagogie, tu affirmais que «les études sur les élèves [de l'enseignement professionnel], leur morphologie sociale, leurs comportements et leurs représentations, qui étaient privilégiées dans les années 1960-1970, semblent peu prisées aujourd'hui ». Comment expliquer ce désintérêt des chercheurs pour l'enseignement technique et professionnel, dont tu as souligné les « coûts en connaissance » dans un article paru en 2005 dans la revue Éducation et Sociétés? Voistu aujourd'hui des changements par rapport à ton bilan de 2000, sachant que Pierre Caspard en 2003, revenant lors d'un colloque à Villeneuve d'Ascq sur cette question, parlait désormais d'une histoire de la formation professionnelle devenue «carrefour » plutôt que chantier déserté ?

Il faut à nouveau revenir sur le glissement sémantique pour voir de quoi on parle. "Le chantier déserté ", selon l'expression déjà citée de Pierre Caspard, concernait essentiellement l'enseignement professionnel, ses institutions, son public, ses enseignants, ses modes de socialisation. Aujourd'hui la notion de formation professionnelle est devenue centrale et déborde largement le cadre scolaire corrélativement à l'accroissement de l'apprentissage qui n'est plus attaché aux savoirs de métiers et aussi à la formation en alternance qui s'étend à toute la hiérarchie des diplômes au-delà du baccalauréat. Plutôt que de tenter de mesurer l'extension des modes d'acquisition des diplômes peut-être vaudrait-il mieux examiner les changements engendrés par l'inscription des problèmes de l'emploi et de l'économie au cœur des politiques éducatives, la redéfinition des missions de l'appareil scolaire eu égard au développement d'une «formation tout au long de la vie » et, plus généralement, une perte de l'autonomie qu'avait conquise l'école tout au long de son histoire.

L'idée de formation synonyme de bien universel a fini par être partagée après un long travail de persuasion. Ainsi, bien que construites à des fins techniques, âprement discutées par les représentants du monde professionnel, les nomenclatures de formation sont maintenant utilisées par des organismes tels que le CEREQ, l'INSEE et les services d'études statistiques et de prospective du ministère de l'Éducation nationale. Elles ont présidé à des représentations durables du monde social et se sont 
progressivement imposées comme catégories de perception communes qui guident les politiques publiques mais aussi les comportements individuels. La pérennité de ces nomenclatures traduit la force des représentations et des croyances collectives qui les sous-tendent et résultent de la convergence d'actions menées, durant les années 1960-70 dans différents lieux de la société et dont le Plan a fait la synthèse. Autant de faits qui sont à l'origine de cette expression nominale « la relation formation-emploi » aujourd'hui assimilée à un nom commun désignant une chose établie.

On ne soulignera jamais assez combien les différents lieux où se mettaient en place des actions de formation continue, ont été simultanément des lieux de réflexion et d'expérimentation pédagogiques et donné naissance à des principes qui ont ensuite été codifiés et étendus à la formation initiale, voire, d'une manière progressive, à tous les segments de l'appareil scolaire et à l'université elle-même.

Le modèle pédagogique des compétences représente peut-être l'exemple le plus éloquent de ce changement pédagogique qui a pénétré l'institution scolaire depuis la publication, en 1991, de la "Charte des programmes", ensemble de principes directeurs selon lesquels les contenus d'enseignement de l'école primaire au lycée doivent être redéfinis; dans ce texte, la notion de compétences est constamment sollicitée.

L'éducation et la formation ont été, l'une et l'autre, construites comme des instruments et des piliers d'un changement d'envergure mais en suivant des orientations différentes. L'éducation a été pensée comme un vecteur d'un futur à faire advenir par le registre politique tandis que la formation est, depuis ses débuts, plus fortement arrimée au régime économique. L'éducation ainsi que le lieu où elle s'accomplit, l'école, se sont constitués en France, au terme de conflits qui leur ont imprimé des marques de neutralité et laïcité, lesquels sont toujours objet de mobilisations sociales pour défendre des points de vue contre d'autres. La formation est une activité où la coopération entre acteurs se réalise sans heurts importants pour aboutir à un accord. Cet accord s'est construit, pour une part, dans l'ambivalence mais aussi dans une acception de l'intérêt général associé à la définition d'une économie compétitive, d'une «modernisation » de la société, de la mise en place d'institutions de participation et de dialogue social dans un contexte de recherche de réduction des conflits sociaux et politiques.

La mise en perspective des transformations sociales auxquelles participent les politiques d'éducation et de formation s'est faite en tâtonnant par des collectifs de chercheurs français et européens. Les titres de mes publications illustrent cette errance dans l'analyse historique et comparative: Éducation et travail, en GrandeBretagne, Allemagne et Italie ${ }^{42}$, «La formation permanente, entre travail et citoyenneté » (numéro spécial d'Education permanente, 2001, n¹49)

Je laisserai aux collègues plus jeunes le soin de discuter cette interprétation et d'apporter des réponses plus précises à cette question du basculement d'un domaine désertique à un carrefour multidirectionnel.

QUESTION : En quoi ton expérience de femme sociologue dans un univers sociologique où les figures masculines dominaient (et s'imposaient) a nourri ta réflexion à ce sujet? Comment as-tu intégré (ou pas) et à mesure de tes travaux et publications, la question des rapports sociaux de sexe et du genre? Peux-tu identifier les mécanismes, questions ou 
rencontres ou réseaux de chercheurs et chercheuses qui t'apparaissent sur ce thème les plus stimulants? En quoi cette perspective a pris sens (ou non) dans tes recherches sur l'enseignement professionnel?

Je dois avouer que j'ai, très tardivement, pris conscience des rapports de domination de genre dans l'exercice de la recherche. Les rapports de classe m'étaient évidents, ils étaient premiers pour moi. J'attribuais tous les obstacles rencontrés aux conditions sociales. Lorsque je suis entrée au CNRS j'avais pour rapporteur R. Boudon qui m'a soutenue sans jamais faire référence d'aucune manière ni à ma condition de femme, ni à mon orientation intellectuelle. Il m'a simplement dit «vous travaillez avec Viviane Isambert qui est une durkheimienne. Venez me voir si vous voulez entendre un autre son de cloche ». P. Naville, mon directeur de thèse, m'a accueillie comme une disciple possible parce que, disait-il, j'étais un cas sur $1000 \mathrm{vu}$ mes origines ! Apparemment rien ne me renvoyait alors à ma condition de femme. A posteriori, je pense que la discipline de travail que je m'imposais a fait reconnaître que j'étais à ma place.

Néanmoins j'ai vécu quelques rares agressions verbales de collègues masculins lorsque je me trouvais en position de pouvoir (bien dérisoire) comme d'être à l'initiative de recherches collectives et, de ce fait, responsable de leur déroulement ou encore après des soutenances de thèse lorsque mon jugement déplaisait au directeur de celle-ci.

J'ai éprouvé une autre expérience discriminante lors de la réalisation de la mission officielle, que m'avait confiée le Secrétariat à l'enseignement technique et professionnel en 1990, peu après la proclamation du mot d'ordre par J.-P. Chevènement, alors ministre de l'Éducation nationale « $80 \%$ d'une classe d'âge au niveau bac » (évoquée plus haut). L'interprétation de cette commande éminemment ambivalente, s'est faite à partir d'un lourd travail d'investigation quantitative (assistée par des collègues du CEREQ et de la DEP) et d'enquêtes auprès de chefs d'entreprises, de représentants d'associations professionnelles patronales, de décideurs dans la haute administration de l'Éducation nationale et dans les régions, tous des hommes investis d'une autorité qu'ils exerçaient avec force dans des milieux masculins. Moi femme, sociologue, de petite taille j'étais a priori dépourvue de toute légitimité et regardée des pieds à la tête avec un air interrogateur. Autant de situations qui, paradoxalement, m'ont donné le sentiment d'être une sorte de porteparole de ceux que je pressentais victimes de cette politique.

Forte de mon indépendance, éprise d'égalité, sensible à l'humiliation, je me suis posée en égale face à ceux que je percevais comme des adversaires. Aux représentants des petites et moyennes entreprises qui me félicitaient d'avoir montré le bien-fondé d'une formation professionnelle sanctionnée par le CAP, je répondais qu'ils contribuaient à sa dévalorisation par les conditions sociales du travail manuel et les bas salaires concédés aux ouvriers et aux employés. Aux représentants de l'UIMM (Union des Industries métallurgiques et minières) qui affirmaient que les entreprises de ce secteur ne recruteraient plus qu'au niveau du BTS, je rappelais les objections de celles qui avaient renvoyé leur carte d'adhésion ainsi que l'opposition des entreprises des régions d'Alsace et de Lorraine confrontées aux entreprises allemandes qui recrutaient les élèves et apprentis titulaires d'un CAP sur le marché du travail des zones frontalières. De fait, nombre de sections formant aux métiers de la mécanique ont été supprimées qui sont aujourd'hui très demandées dans divers secteurs 
industriels tels que la construction et réparation navale, l'aéronautique, l'automobile, l'industrie nucléaire. Parmi eux, les métiers de la chaudronnerie et de la soudure alors considérés comme dépassés s'avèrent être les plus déficitaires ${ }^{43}$.

Ainsi j'ai pu lever les barrières de genre dans ce type de situation.

Dans le monde scientifique, j'ai remarqué, avec d'autres, que dans des situations de prise de décision - comités scientifiques, jury de recrutement, comités de rédaction de revues - la parole d'une femme ne pesait pas le même poids que celle d'un homme. La première n'était souvent entendue que si l'autre la reprenait en se l'appropriant.

Dans des relations d'enquête, j'ai compris que la première condition à remplir pour lever les préjugés est sans doute de respecter l'autre. J'en fis l'expérience (ainsi que C. Agulhon et A. Poloni) dans la recherche sur les enseignants professionnels tous, à une seule exception, étaient de sexe masculin, Confrontées à une défiance spontanée à l'égard des "dames du CNRS ", mises à l'épreuve de multiples façons lors des premiers entretiens, nous avons dû faire la preuve de notre intérêt aux problèmes techniques et d'une connaissance minimale pour obtenir des informations, notamment sur les pratiques de transmission des savoirs techniques et professionnels. Pour cela, nous avons dû acquérir chacune à notre manière une certaine familiarité avec les machines, avec les définitions des principales notions techniques, en bref, apprendre à nous servir d'une manière approximative du langage technique. Nous pouvions alors questionner tout en laissant voir notre ignorance qui était acceptée au prix de notre bonne volontét ${ }^{44}$.

Paradoxalement, notre statut et notre sexe qui constituaient des handicaps pour aborder certains registres de questions devenaient des avantages lorsqu'il s'agissait de recueillir des informations sur les questions jugées d'ordre privé, comme celles relatives aux parents et aux enfants par exemple, ou sur les questions psychologiques extrêmement vives dans la mémoire de ces enseignants qui nous ont confessé avoir souffert dans leur corps pour assurer leur passage du statut d'ouvrier à celui de professeur. 
Images 6 et 7 : Piquage

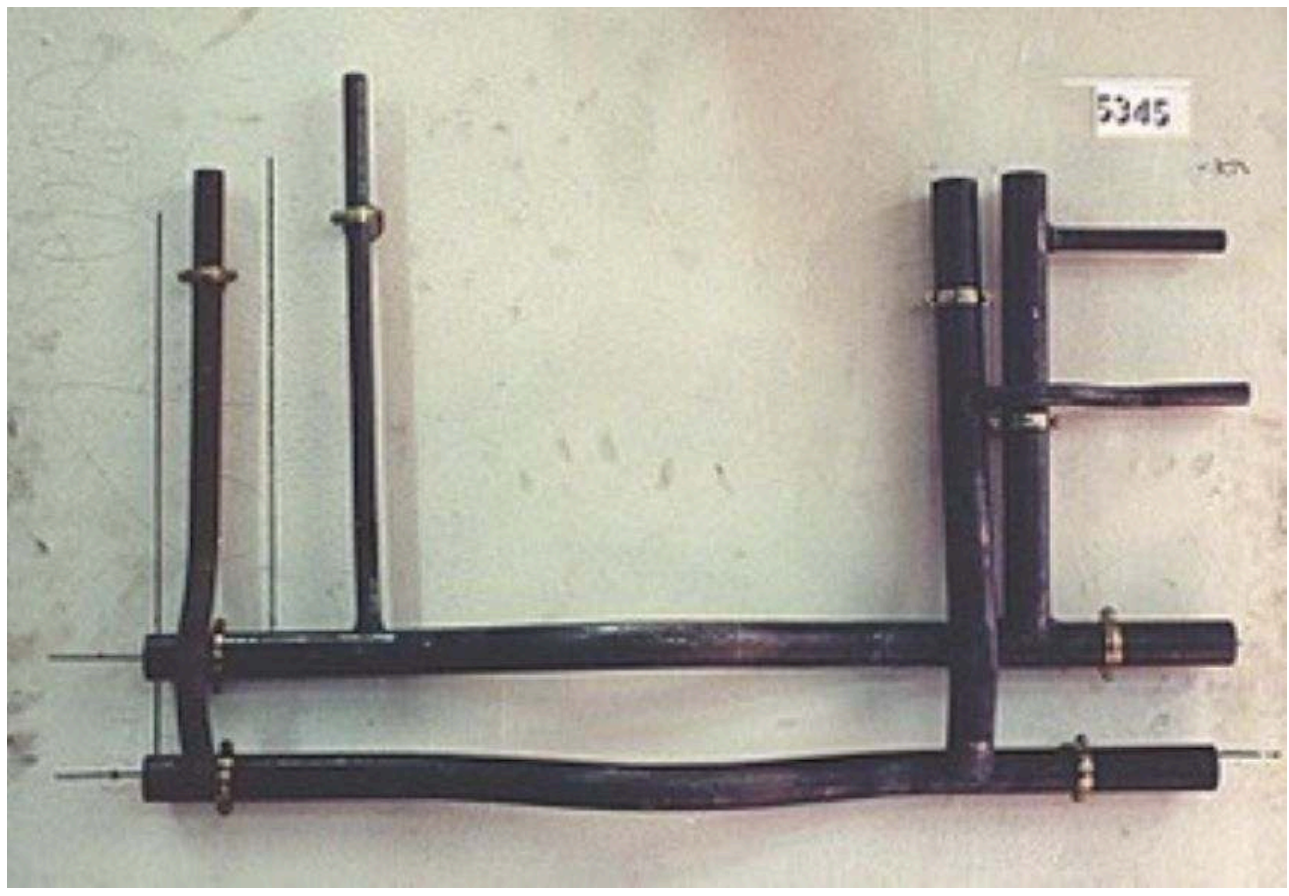

Image 7

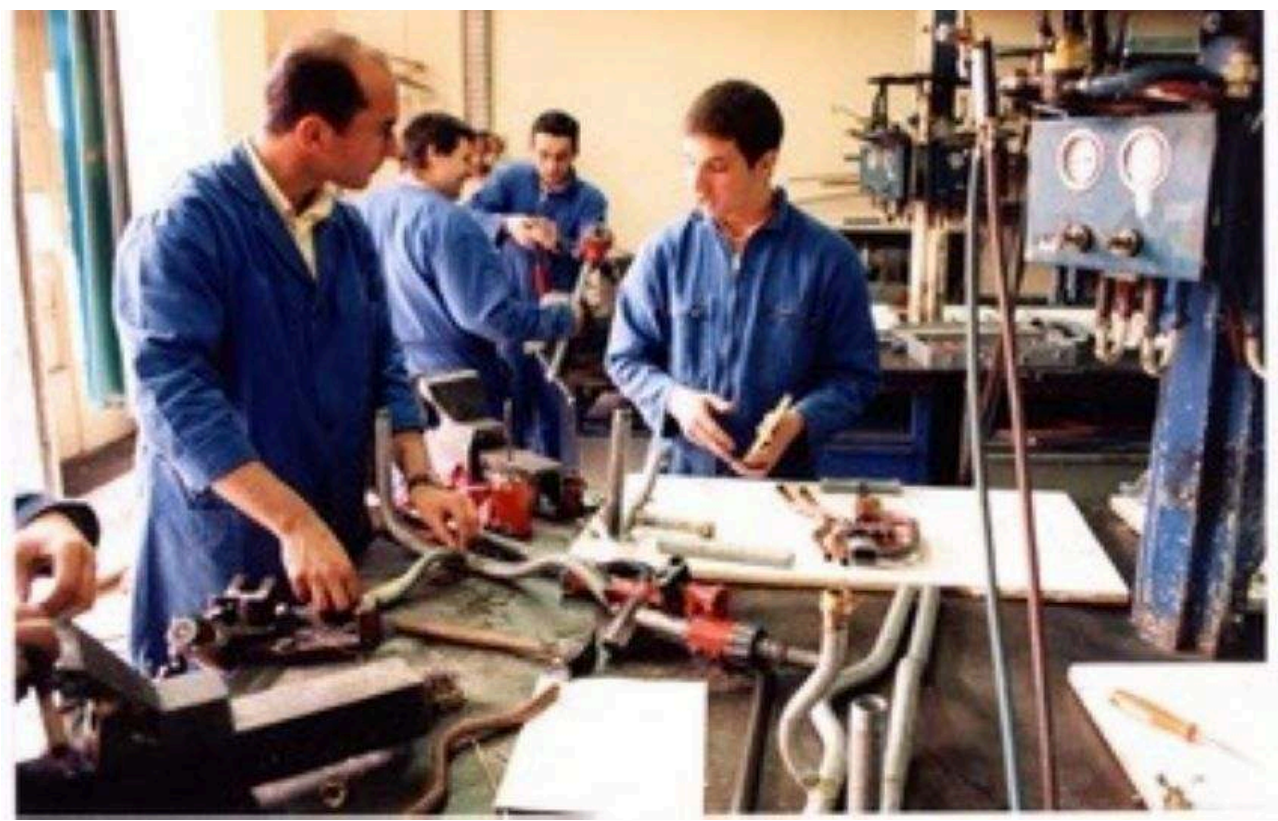

(CDaniel Tacaille

Pour clore, je dirai que je n'ai pas le sentiment d'avoir subi d'entraves à ma carrière de chercheuse, tout au plus éprouvé des préjugés dont je me suis défendue et deux ou trois humiliations qui en réveillaient d'autres très anciennes desquelles je n'avais pu me défaire sur le champ, faute de disposer de l'équipement nécessaire. Je dois pourtant reconnaître que j'effaçais les rapports de domination de genre dans l'enseignement professionnel, dans la recherche, voire d'une certaine façon dans le travail en général en donnant toute leur importance aux rapports de domination de 
classe. Vous qui me demandez cet entretien, et les chercheur-es auxquels vous êtes associés ainsi que des ancien-es doctorant-es remédiez à cette part d'ombre et c'est une grande avancée en sociologie.

QUESTION : Au cours de ton travail de recherche, dont une partie s'est fait à partir d'archives, tu as dû croiser des images (photos, films, etc.) relatives à l'enseignement technique ou professionnel ou au travail. Quel était ton attitude face à ce matériau que les sociologues ont souvent évité ou méprisé ? T'y arrêtais-tu ? En quoi te "parlaient-elles »? En stockaistu? Et si oui avec quel éventuel projet ? Certaines t'ont-elles particulièrement marquée? Pourrais-tu en présenter quelques-unes et les commenter?

Les images sont plutôt rares dans les archives que j'ai dépouillées hormis celles de photos de classe révélant des élèves aux visages et aux corps plus âgés que ceux d'aujourd'hui. Dans les années 1970-80 l'image avait peu de place dans les comptes rendus d'enquête et encore moins dans les publications. Bourdieu insistait beaucoup sur la description des lieux de vie, des corps, de l'habillement, du langage qui nourrissait le récit mais nous n'avions pas d'appareil pour les photographier. L'image n'avait pas la place qu'elle a aujourd'hui où elle envahit tous les moments de la vie.

Par contre, j'ai gardé en mémoire des images mentales d'enseignants dans leurs ateliers, face à leurs élèves et à moi-même. Me revient celle où ils m'attendaient derrière les grandes vitres de l'atelier et m'accueillaient ensuite avec ironie « Mais vous savez conduire!». Me reste également celle d'un homme ancien ouvrier qualifié, devenu enseignant parce que sa carrière était bloquée dans l'usine et qui a souffert dans son corps et sa dignité sa conversion au métier enseignant : s'exerçant à parler aux élèves face à un miroir en évitant les tics de langage que les élèves ne manquaient pas de mimer. Les images "matérialisées » qui me restent en tête sont celles de films destinés à être projetés, où le travail est idéalisé, à l'exemple de l'ouvrier sidérurgiste face aux dangers du métier que l'homme va maîtriser, soient des images édifiantes et aliénantes tout à la fois. 
Image 8 : Couverture de l'ouvrage La sociologie du travail en France : enquête sur le travail des sociologues, 1950-1990, Paris, La Découverte, 2011.
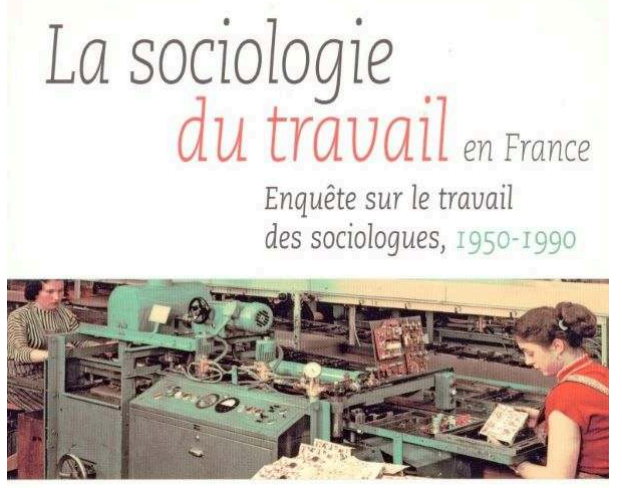

Lucie Tanguy

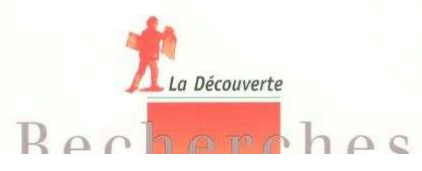

L'image s'introduit aujourd'hui dans les ouvrages à caractère universitaire parce qu'elle possède des vertus propres. Les éditeurs y ont fréquemment recours notamment pour illustrer les couvertures. C'est ainsi que mon ouvrage sur «La sociologie du travail» est agrémenté par l'image d'ouvrières en téléphonie ${ }^{45}$. Proposée, parmi d'autres, par l'éditeur, je m'y suis ralliée sans réserve d'autant plus qu'elle montrait des femmes au travail, type de travail auquel je ne m'étais pas intéressée. 
Image 9 : Couverture de l'ouvrage Enseigner l'esprit d'entreprise à l'école : le tournant politique des années 1980-2000 en France, Paris, La Dispute, 2016.

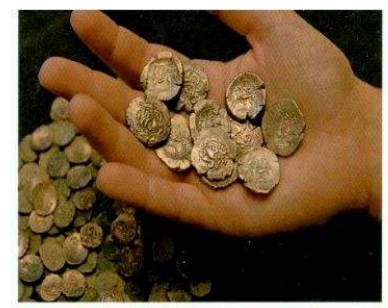

Lucie Tanguy

\section{Enseigner}

l'esprit d'entreprise

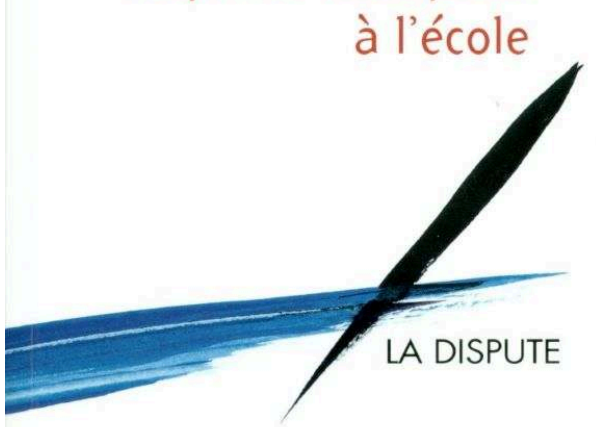

Le choix de l'image apposée sur la couverture de cet autre ouvrage intitulé « Enseigner l'esprit d'entreprise à l'école ", qui représente une main emplie de pièces de monnaie, répondait à deux exigences d'ordre différent : pouvoir être reproduite gratuitement et évoquer le sens que je voulais donner à l'analyse développée dans le corps $d u$ livre $^{46}$. On a pu me reprocher de réduire l'entreprise au gain d'argent, critique dont la justesse est indéniable. Mais dans la mesure où il m'importait de montrer que l'habilitation des entreprises à contribuer à l'éducation de la jeunesse, au moyen d'associations les représentant, avait pour intention d'inculquer, non pas tant l'apprentissage de connaissances, que des valeurs et des comportements culturels propres à ce que Max Weber appelle l'esprit du capitalisme ${ }^{47}$. Le capitalisme ne désigne pas chez cet auteur un système économique et social fondé sur l'appropriation des moyens de productions comme chez Marx. Il se définit plutôt comme une rationalisation instrumentale des activités sociales qui exige la formation de schèmes de pensée devant pénétrer toutes les sphères de la vie collective et individuelle.

QUESTION : Lorsque tu regardes ton travail de sociologue, même si tu conduis encore des recherches et publies encore, as-tu des regrets? Des choses que tu ferais ou aurais fait autrement ? Des renoncements que tu regrettes?

«Bilan » est un mot du commerce qui se définit comme le rapport entre les actifs et les passifs. Impossible de faire de telles opérations sur les phénomènes sociaux qui ne se laissent pas réduire à des valeurs numériques.

De toute façon, évaluer les éventuelles avancées de la recherche en sciences sociales, auxquelles on a participées ou que l'on a impulsées, ne peut se faire qu'à l'échelle collective après avoir réuni les conditions nécessaires à cet exercice. Exercice 
périlleux dans un domaine où la diversité des points de vue se côtoie, où les objets glissent suivant les problèmes sociaux du moment. Il reste, me semble t-il, une posture d'esprit que j'appellerai engagement qui n'est en rien une simple dénonciation. L'engagement est, il est vrai, une notion ambivalente qui implique une critique d'un ordre établi au nom d'un avenir définissable à partir d'objectifs historiques et qui s'accompagnent d'un processus d'émancipation. J'ai tenu cet engagement en me référant aux objectifs fixés par des penseurs progressistes, comme ceux cités plus haut, qui, au lendemain de la seconde guerre mondiale, proposaient de transformer les contenus d'enseignement de sorte à s'affranchir de cette vision aliénante qui consistait à définir l'universalisme essentiellement par l'« enseignement général ». À l'encontre, ils avançaient l'idée d'un enseignement unissant l'apprentissage d'un métier à une émancipation culturelle. Mais l'extension de la scolarisation du dernier demi-siècle s'est effectuée en préservant la prééminence de l'enseignement général et en dépréciant d'une manière continue l'enseignement professionnel malgré l'inflation des discours appelant à le valoriser puis, récemment, à lui substituer l'apprentissage en entreprise. Peu étudié, cet enseignement est pourtant aujourd'hui comme hier, l'objet des transformations les plus radicales et les plus politiques. L'histoire a montré que les changements qui les affectent sont souvent annonciateurs de ceux qui s'étendent ensuite aux autres segments du système éducatif. Un enseignement assemblant la formation à un métier, à une émancipation culturelle demeure néanmoins un horizon d'attente pour tous ceux qui sont épris de justice sociale.

L'un des regrets qui me tenaillent toujours est l'effacement de controverses ouvertes, argumentées, au profit d'un évitement qui stérilise l'avancée des connaissances et des transformations possibles. Mes analyses sur l'enseignement professionnel, appuyées sur ce que celui-ci contenait potentiellement opposées à celles qui voyaient dans cet enseignement un apprentissage à la discipline, à l'obéissance aux règles de l'ordre existant n'ont jamais donné lieu à quelque confrontation que ce soit. Selon la thèse développée dans L'ordre des choses, le CET joue un rôle de redressement moral autant que de formation technique: compenser les lacunes d'une prime éducation "relâchée ", apprendre aux adolescents les usages de base en matière d'espace (une place pour chaque chose), de temps (un temps pour chaque chose), de conformité instrumentale (utiliser les outils à bon escient, verbale (appeler les choses par leur nom), morale et hygiénique (se laver, faire du sport), être franc, loyal, courageux, volontaire etc. À quoi les élèves opposent des réactions de contre-acculturation caractéristique : fugues, chahut sauvage de type mutinerie, langage ordurier, jeux violents etc.. Un retour sur cette thèse aujourd'hui susciterait, sans doute, de sévères critiques parce qu'elle porte exclusivement sur le registre de la culture, du rapport entre culture technique et professionnelle et culture "culturelle ", " "générale ", « dominante » niant les savoirs qui y sont rattachés.

Ce type d'analyse, ancrée dans la théorie de $L a$ reproduction $^{48}$, repose sur une conception de la connaissance et des rapports entre connaissance et action très discutable. Elle postule qu'il existe un ordre unidimensionnel des savoirs, selon lequel les savoirs techniques seraient une application des savoirs scientifiques et les savoirs professionnels une application des savoirs techniques, autrement dit elle s'appuie sur une conception qui nie l'exigence de registres spécifiques de savoirs et qui empêche l'incorporation de l'enseignement technologique dans la culture scolaire. Une telle 
conception s'oppose à la reconnaissance de différentes formes d'excellences et nie la rationalité de l'action dans le travail. Tels sont les principes qui m'ont guidée dans mes recherches. Près de vingt ans séparent ces deux points de vue, construits dans des contextes différents et qui, on le voit, s'opposent mais ne se confrontent pas.

Une réflexion critique serait tout aussi nécessaire aujourd'hui pour lever l'opacité sémantique contenue dans le caractère polymorphe de cette expression « une culture commune " promue par un courant de pensée pour rendre l'école égalitaire.

Autre déception, ma faible connaissance des langues étrangères (celle de ma génération), qui m'a empêchée de mener des comparaisons internationales, méthodes de recherche qui s'imposent aujourd'hui à l'heure de la mondialisation. Celles-ci nécessitaient des investissements intellectuels et financiers que nous ne possédions pas. Les rares tentatives réalisées Éducation et travail, en Grande-Bretagne, Allemagne et Italie 1995 (traduit en anglais) réalisées le plus souvent en collaboration avec d'autres collègues (A. Jobert, C. Marry) ou encore L'école et l'entreprise, l'expérience des deux Allemagnes (avec Annick Kieffer) sont restées à l'état de délimitation de thématiques privilégiées ici où là, permettant une interconnaissance d'un milieu de recherche mais non une connaissance concrète des phénomènes eux-mêmes.

J'ajouterai à nouveau cet embarras causé par les discontinuités qui s'opposent à la cumulativité des connaissances qui pourrait s'établir sur une classe d'objets empiriquement identifiées, sur les modes de recherche, les conceptions de la preuve, les critères d'objectivité etc. Mais le temps passant, je me suis libérée de la question du pourquoi et de cette volonté obsessionnelle de «faire science » au sens normatif du terme.

Au final, je dirai que je me définis comme un passeur (mettre le mot au féminin serait le dénaturer) qui essaie de transmettre les leçons de son expérience d'un métier qui doit conjuguer des exigences contraires : celles afférentes à l'autonomie de la pensée et celles liées à l'engagement dans la société de son temps. Examiner les recherches réalisées et leurs résultats est un préalable à toute perspective sociologique. Transmettre les leçons de son expérience n'a par conséquent d'intérêt que s'il se fait sur le mode d'une analyse réflexive et non sur celui du témoignage. Faute de quoi, je l'ai montré, un chercheur, une chercheuse peut accompagner à son insu une politique dont il ou elle n'avait pas déchiffré le sens ou, à l'inverse, montrer qu'elle accentue les inégalités sociales. Je crois avoir montré la fécondité d'une sociologie relationnelle à propos de l'émergence puis l'inflation de l'usage de la notion de compétences et des changements qu'elle désignait dans la sphère éducative et celle du travail. La traduction de cet ouvrage au Brésil s'est faite sur le constat d'une diffusion de cette notion dans ce pays, qui témoigne également de changements transversaux à cette société.

Au terme de ce parcours, je dirai que mon engagement dans le monde social a présidé au choix et au découpage de mes objets de recherche, au mode de constitution et de traitement des données et aux formes de démonstration. Cette posture intellectuelle associée à une démarche sociohistorique et à la combinaison de différents niveaux d'analyse sous-tendent l'investigation menée dans mon dernier ouvrage. Elle souligne les principales transformations en cours, encore en germe dont il reste à approfondir la signification: un processus de délitement du service public, un glissement de la mission première de l'école, former un-e citoyen-e sachant exercer 
un jugement critique à celle d'un sujet essentiellement adapté aux impératifs économiques, une substitution de valeurs collectives au premier rang desquelles l'égalité à des projections de réussite individuelle ${ }^{49}$. Au fil des années, j'ai tenté de tenir un double engagement dans la production de connaissances et dans l'action.

\section{NOTES}

1. Voir pour une approche synthétique, Benjamin Stora, Histoire de la guerre d'Algérie, $4{ }^{\text {ème }}$ édition, Paris, La Découverte, 2004.

2. Cf. Pierre Bourdieu (dir.), Un art moyen. Essai sur les usages sociaux de la photographie, Paris, éditions de Minuit, 1965.

3. L'EPRASS a été créé par le $\mathrm{VI}^{\circ}$ section de l'EPHE et des chercheurs du CNRS à la rentrée universitaire en 1965. Cet enseignement concernait quatre disciplines : la sociologie, la plus nombreuse (30 personnes au maximum), l'ethnologie, la psychologie, et la linguistique.

4. À l'époque, l'entrée au CNRS s'effectuait sur concours et présentation d'un projet de recherche mais le rapport entre le nombre de postes affichés et le nombre de candidats n'était en rien comparable à celui d'aujourd'hui pas plus que ne l'étaient les exigences en matière de certifications académiques et de publications dans des revues scientifiques. Sans aller plus avant sur les modalités de recrutement et de fonctionnement de la recherche au CNRS, on rappellera qu'en 1965, on comptait seulement 75 chercheurs en sociologie, Jacques Lautman, «Chronique de la sociologie française après 1945 ", Science théorique de l'opinion publique, hommage à J. Stoetzel, Paris, Retz, 1981.

5. Lucie Tanguy, «L'État et l'école. L'école privée en France », Revue française de sociologie, 1972, 13-3, p. 325-375. Sur la loi Debré de 1959, voir aussi Bruno Poucet (dir.), L'État et l'enseignement privé : l'application de la loi Debré (1959), Rennes, PUR, 2011.

6. Lucie Tanguy, Enseigner l'esprit d'entreprise à l'école. Le tournant politique des années 1980-2000 en France, Paris, La Dispute, 2016

7. Lucie Tanguy, Le capital, les travailleurs et l'école: l'exemple de la Lorraine sidérurgique, Paris, François Maspero, 1976.

8. Monique Segré-Brun, Lucie Tanguy, «Quelle unité d'analyse retenir pour étudier les variations géographiques de la scolarisation? », Revue française de sociologie, numéro thématique « Sociologie de l'éducation ", 1967, 8, 1, p.117-139.

9. Analysant l'évolution de la scolarisation entre 1969 et 1974 en prenant pour base 100 l'état de celle-ci en 1969, on constate que : la filière professionnelle en CET connait l'évolution la plus faible, passant de l'indice 100 à l'indice 103 ; la filière dite de relégation (désignant le préapprentissage et l'apprentissage), elle, passe de l'indice 100 à l'indice 157, quant aux lycées, tout réservés qu'ils étaient, s'étendaient de l'indice 100 à l'indice 116. Je soulignais que cette évolution n'était pas le produit d'un mouvement spontané, ni la résultante d'une somme de comportements individuels désignés sous l'étiquette "mépris de l'enseignement technique ", mais l'effet de la politique de l'État alors définie dans le cadre de la carte scolaire. Ces processus se déroulaient sur la base de changements dans la division sociale du travail dont une déqualification massive du travail ouvrier : de 1954 à 1968, le nombre d'ouvriers spécialisés est passé de 1815625 à 2650380 alors que celui des ouvriers qualifiés est passé, lui, de 2837442 à 
2506 180. Lucie Tanguy, La carte scolaire, instrument d'une politique d'État, Université Paris V, UER Sciences de l'Éducation, 28 rue Serpente 75006.

10. Daniel Tacaille a été professeur de psycho-pédagogie à l'École nationale normale d'apprentissage de Paris Nord de 1979 à 1991 puis de 1991 à 2001. Cette discipline fut introduite dans la formation des maîtres par les pionniers de cette institution pour faire se rencontrer "production et culture ». Nous le remercions pour les clichés qu'il a bien voulu nous confier à la demande de Lucie Tanguy.

11. Voir Le capital, les travailleurs et l'école, ch.11, Les CET, un enjeu politique. Patrice Pelpel, Vincent Troger, Histoire de l'enseignement technique, Paris, Hachette, 1993, des filières sélectives, p. 97.

12. Le technique en lutte, CET, lycées techniques, c'est déjà l'usine! Supplément à Libérons l'école, №1: « $40 \mathrm{~h}$ par semaine c'est trop. C'est énorme et ça ne laisse pas le temps de vivre et de penser un peu. Pourquoi 40h par semaine? Quand on voit que les copains qui bossent n'utilisent pas le dixième des conneries qu'on leur a apprises, mais qu'ils font un boulot abrutissant toujours le même; quand on voit que moins de la moitié des élèves qui sortent des CET bossent dans la branche qu'ils avaient étudiés, on se dit 40heures par semaine, ce n'est pas tant pour nous donner une qualification professionnelle que pour NOUS HABITUER AUX HORAIRES D'USINE EPUISANTS qui engraissent les patrons, mais qui nous empêchent de vivre et penser par nousmêmes en dehors du bourrage de crâne des profs et de la télé ".

D'autres journaux témoignent de la place de l'enseignement technique en mai 68 et par la suite : «CET, Lycées, Facultés / Tous unis dans la lutte! A bas l'embrigadement de la jeunesse, A l'Armée, A l'école! Le CET c'est déjà l'usine ! CET-Caserne, Y'en a marre ». Technique rouge, Journal de la Ligue communiste et des cercles rouges du technique, 1973, n³. "CET-Casernes Ras-l'bol», «Faire de la politique pour ne pas être victimes des politiciens », Ceux du technique, Journal des CET édité par Lutte ouvrière, $1973, \mathrm{n}^{\circ} 2$.

13. Jean-G. Padioleau, « Raymond Boudon, L'analyse mathématique des faits sociaux », Annales, Économies, Sociétés, Civilisations, 1969, 24-4, p. 947-948.

14. Raymond Boudon, Paul Lazarsfeld, Le vocabulaire des sciences sociales : concepts et indices, Paris, La Haye, Mouton\&Co, 1965, Raymond Boudon, Paul Lazarsfeld, L'analyse empirique de la causalité : choix de textes, Paris, La Haye, Mouton \&Co, 1966.

15. Jean-Daniel Reynaud, « Sociologie et « raison dialectique » ", Revue française de sociologie, 1961, 2-1, p. 50-66.

16. Voir Emmanuel Droit, Vers un homme nouveau ? L'éducation socialiste en RDA : 1949-1989, Rennes, PUR, 2009.

17. Notes et études documentaires $n^{\circ} 4669-4670$, La Documentation française.

18. Mona Granato, Gilles Moreau, Les défis de l'apprentissage en Allemagne, Formation Emploi, $\mathrm{n}^{\circ} 146,2019,2$, p. 7-28.

19. Basil Bernstein, Class, codes and control, London, Routledge and Kegan Paul, 4 volumes, 1971, 1973, 1975, 1990 ; Basil Bernstein, Langage et classes sociales: codes socio-linguistiques et contrôle social, Paris, éditions de Minuit, 1975 ; M.E.D. Young, Knowledge and Control : New directions for the sociology of Education, London, Collier-Macmilan, 1971; Jérome Deauvieau, Jean-Pierre Terrail (textes présentés et choisis par), Les sociologues et la transmission des savoirs, dix ans après, Paris, La Dispute, 2017.

20. Lucie Tanguy, "Savoirs et rapports sociaux dans l'enseignement secondaire en France", Revue française de sociologie, 1983, 24-2, p. 227-254; Lucie Tanguy, «Les savoirs enseignés aux ouvriers ", Sociologie du travail, 1983, 25-3, p.336-354 ; Lucie Tanguy, Catherine Agulhon, Françoise Ropé, «L'enseignement du français en LEP, miroir d'une perte d'identité », Études de linguistique appliquée, $\mathrm{n}^{\circ} 54, \mathrm{p} .19-38$.

21. Les IUFM créés en 1990, étaient des établissements de formation des professionnels de l'enseignement public (professeurs du premier et du second degrés, conseillers principaux 
d'éducation). Ils ont été remplacés par les écoles supérieures du professorat et de l'éducation (ESPE), puis par les Instituts nationaux supérieur du professorat et de l'éducation (INSPE).

22. Recyclés est ici synonyme de formés.

23. Lucie Tanguy, L'enseignement professionnel en France: des ouvriers aux techniciens, Paris, PUF, 1991

24. Claude Grignon, L'ordre des choses: les fonctions sociales de l'enseignement technique, Paris, éditions de Minuit, 1971.

25. Lucie Tanguy, « La mise en équivalence de la formation avec l'emploi dans les $\mathrm{IV}^{\mathrm{e}}$ et $\mathrm{V}^{\mathrm{e}}$ Plans (1962-1970) », Revue française de sociologie, 43-4, 2002, p. 685-709.

26. Pierre Caspard, «Un chantier déserté : l'histoire de l'enseignement technique », Formation Emploi, $\mathrm{n}^{\circ}$ spécial, L'enseignement technique et professionnel, repères dans l'histoire (1830-1960), 1989, n²7-28, p. 193-197.

27. Lucie Tanguy, Histoire et sociologie de l'enseignement technique et professionnel : un siècle en perspective, Revue française de pédagogie, 2000, n¹31, p. 97-127.

28. Viviane Isambert-Jamati, Crises de la société, crises de l'enseignement : sociologie de l'enseignement secondaire français, Paris PUF, 1970 ; id. Les savoirs scolaires / enjeux sociaux des contenus d'enseignement et de leurs réformes, 2éd. Paris, L'Harmattan, 1995 (1990).

29. Voir en dernier lieu Laurent Gutierrez et Pierre Kahn (dir.) Le Plan Langevin-Wallon : histoire et actualité d'une réforme de l'enseignement, Nancy, Presses Universitaires de Nancy-Editions Universitaires de Lorraine, 2016.

30. Georges Friedmann, Pour l'unité de l'enseignement : humanisme du travail et humanités, Cahier des Annales, n5, Paris, Librairie Armand Colin, 1950.

31. Lucie Tanguy, La sociologie du travail en France, Enquête sur le travail des sociologues, 1950-1990, Paris, La Découverte, 2011.

32. Lucie Tanguy, Les Instituts du travail: la formation syndicale à l'université de 1955 à nos jours, Rennes, PUR, 2006.

33. Fernand Braudel, « Histoire et sociologie », dans Georges Gurvitch (dir), Traité de sociologie, Paris, PUF, 1958, repris dans Fernand Braudel, Ecrits sur l'histoire, Paris, Flammarion, 1969, p. 97-122.

34. Pierre Naville, École et société, Paris, Librairie Marcel Rivière, 1959 ; Pierre Naville, Théorie de l'orientation scolaire et professionnelle, Paris, Gallimard, 1945, 1972 ; Pierre Naville, La formation professionnelle et l'École, Paris, 1948. À l'occasion de cet exercice, je retrouve une publication oubliée portant sur les traces laissées par P. Naville sur mes perspectives de recherche dans l'hommage réalisé par Michel Éliard, Pierre Naville, La passion de la connaissance, L. Tanguy, «P. Naville, 1942-1960, une sociologie des relations ", Toulouse, Presses du midi, 1996.

35. Françoise F. Laot et Rebecca Rogers, Les sciences de l'éducation, émergence d'un champ de recherche dans l'après-guerre, Rennes, PUR, 2015.

36. Françoise Ropé, Lucie Tanguy (dir.), Savoirs et compétences : de l'usage de ces notions dans l'école et l'entreprise, Paris, L'Harmattan, 1994 (Saberes e competências, Sao Paulo 1996).

37. Norbert Elias, Qu'est-ce que la sociologie ? rééd. La Tour-d'Aigues, éditions de l'Aube, 1996 (1970), p. 73-76.

38. N. Elias, Engagement et distanciation, Paris, Fayard, 1993, chap. 1

39. Lucie Tanguy, « Le sociologue et l'expert, une analyse de cas », Sociologie du travail, 1995, 37-3, p. 457-477.

40. Paris, La Documentation française, 1991.

41. Expression utilisée par Pierre Caspard, dans la postface d'un numéro spécial de la revue Formation Emploi, consacré à « l'enseignement technique et professionnel. Repères dans l'histoire (1830-1960)» : Pierre Caspard, « un chantier déserté : l'histoire de l'enseignement technique », Formation Emploi, n²7-28, 1989, p. 193-197. 
42. Annette Jobert, Catherine Marry, Lucie Tanguy, Education et travail, en Grande-Bretagne, Allemagne et Italie, Paris, Armand Colin, 1995, Annette Jobert, Catherine Marry, Lucie Tanguy and Helen Rainbird (dir.), Education and Work in Great Britain, Germany and Italy, London and New York, 1997 ; Lucie Tanguy, Philippe Casella, Guy Brucy (dir.), « Entre travail et citoyenneté, la formation permanente. France, Allemagne, Suède », Éducation permanente, 2001, n¹49.

43. Voir l'Usine nouvelle, 2-12-2016 et 30-3-2016. Parmi les exemples cités, celui d'EDF, producteur et distributeur d'électricité qui dit ne recevoir que 30 réponses pour une annonce de poste de chaudronnier contre 300 pour un poste administratif.

44. Voir Catherine Agulhon, Arlette Poloni, Lucie Tanguy, Des ouvriers de métiers aux diplômés du technique supérieur: le renouvellement d'une catégorie d'enseignants en lycées professionnels, Paris, Ministère de l'Éducation Nationale, CNRS, 1988.

45. Ouvrières chez Telefunken, 1958.

46. Illustration de couverture : trésor de Laniscat (Côtes d'Armor) 2007. Découvert dans une exploitation agricole de l'âge du fer, le trésor de Laniscat se compose de 58 statères et de 487 quarts de statères. Toutes ces monnaies ont été frappées par le pouvoir osisme au cours de la première moitié du $1^{\mathrm{er}}$ siècle av. J.C., Institut national de recherches archéologiques préventives, RMN-Grand Palais, photo Hervier Paitier.

47. Max Weber, L'Ethique protestante et l'esprit du capitalisme (1904), Plon, Paris, 1967.

48. Pierre Bourdieu, Jean-Claude Passeron, La Reproduction, Paris, éditions de Minuit, 1970.

49. Lucie Tanguy, Enseigner l'esprit d'entreprise dans l'école, op. cit.

\section{AUTEURS}

\section{STÉPHANE LEMBRÉ}

Maître de conférences en histoire contemporaine à l'université de Lille (Institut national supérieur du professorat et de l'éducation) et membre du laboratoire CREHS (université d'Artois). Dans le sillage d'une thèse soutenue en 2011 et qui a donné matière à un livre intitulé L'école des producteurs. Aux origines de l'enseignement technique en France 1800-1940 (Rennes, Presses universitaires de Rennes, 2013), puis d'une synthèse publiée en 2016 (Histoire de l'enseignement technique, Paris, La Découverte, coll. « Repères »), ses travaux portent sur l'histoire de l'éducation et de la formation professionnelle aux $\mathrm{XIX}^{\mathrm{e}}$ et $\mathrm{XX}^{\mathrm{e}}$ siècles, aux échelles nationale et internationale, en prêtant attention aux acteurs (économiques, éducatifs et politiques) comme à la matérialité de l'éducation et de la formation. Dans cette perspective il aborde depuis plusieurs années les images (clichés photographiques et films documentaires) en tant que sources longtemps négligées et pourtant propices, d'une part, à une histoire des représentations, et d'autre part, à une exploration de la matérialité de l'éducation et de la formation.

\section{GILLES MOREAU}

Professeur de sociologie à l'université de Poitiers et membre du GRESCO (Groupe de recherche sociologique sur les sociétés contemporaines). Ses recherches portent sur la formation professionnelle des jeunes, en lycée professionnel et en centre de formation d'apprentis, avec depuis quelques années une attention spécifique portée aux diplômes professionnels. Il a notamment publié Le monde apprenti (Paris, La Dispute, 2003) et co-coordonné plusieurs 
ouvrages en rapport avec ces thématiques dont La société des diplômes (Paris, La Dispute, 2011) et Le CAP, un diplôme du peuple (1911-2011) (Rennes, Presses universitaires de Rennes, 2013). Il a par ailleurs récemment coordonné deux numéros de la revue Formation Emploi consacrés à l'apprentissage en Suisse ( $\left.n^{\circ} 133-2016\right)$ et en Allemagne ( ${ }^{\circ}$ 146-2019). Il poursuit actuellement des recherches sur le CAP et les savoirs professionnels

\section{LUCIE TANGUY}

Chercheure associée au CRESPPA-GTM, CNRS

ltanguy2[at]gmail.com 